\title{
TISSUE CHARACTERIZATION OF
} PROSTATE CANCER USING

\section{QUANTITATIVE ANALYSIS OF LOW FREQUENCY ULTRASOUND \\ by}

ERVIS SOFRONI, B.SC., RyERSON UNIVERSITY,TORONTO,2011

\author{
A thesis \\ presented to Ryerson University \\ in partial fulfillment of the \\ requirements for the degree of \\ Master of Science \\ in the Program of \\ Computer Science
}

(C) Ervis Sofroni 2011 


\section{Author's Declaration}

I hereby declare that I am the sole author of this thesis.

I authorize Ryerson University to lend this thesis or dissertation to other institutions or individuals for the purpose of scholarly research.

*

I further authorize Ryerson University to reproduce this thesis by photocopying or by other means, in total or in part, at the request of other institutions or individuals for the purpose of scholarly research. 


\title{
AbStract TISSUE CHARACTERIZATION OF PROSTATE CANCER USING
QUANTITATIVE ANALYSIS OF LOW FREQUENCY ULTRASOUND
}

\author{
MSc., 2011, Ervis Sofroni, Computer Science, Ryerson University
}

Current accepted methodologies used for detection of the prostate tumor involve measurements of prostate specific antigen (PSA) levels, patient age followed by ultrasound guided biopsies leaving a lot to desire in the ability to correctly identify lesions. Also PSA level test has been shown to produce a high number of false positives leading to unnecessary invasive biopsies. The goal of this thesis is to investigate the use of trans-rectal conventional low frequency $(1-10 \mathrm{MHz})$ ultrasound as a non-invasive imaging modality for the detection of prostate tumors. Currently we are investigating the use of multi parameter spectroscopic analysis of the ultrasound radiofrequency signal in combination with ultrasound elastrographic imaging of the prostate and correlating the results with whole-mount histopathology from radical prostatectomy. Ten patients with prostate cancer prior to surgery were subjected to trans-rectal conventional low frequency ultrasound scans. Parametric maps are generated for each individual spectral parameter. Ratios of disease area versus normal prostatic tissue are identified using low frequency ultrasound and compared with the equivalent ratios obtained from whole-mount histopathology. Preliminary results show that areas of suspected disease identified by spectral parameters correlate with areas of disease presence in the corresponding whole-mount sections. An initial software platform performing visualization of areas of disease based on parametric maps generated from spectral analysis methods was developed. 


\section{Acknowledgments}

I would like to acknowledge the support of all the individuals who assisted and supported me through the years of this research. I would like to acknowledge my supervisor Dr. Alireza Sadeghian for providing me the opportunity to continue my education and reach my goals and without whose support I would not have been able to accomplish this work.

I would also like to acknowledge and thank Dr. Gregory Czarnota for allowing me to conduct research in his laboratory at Sunnybrook Health Sciences Centre, and who provided me with constant guidance and access to data.

I wish to extend my gratitude to Dr. Michael Kolios who as part of my supervisory committee has assisted me in building the fundamental understanding of the physics needed to progress with my research.

Additionally, I would like to thank all the members of the Czarnota lab team and Sunnybrook Health Sciences Centre staff who have provided me guidance and assistance through the years.

I would like to extent my gratitude and thank my colleague and fellow student Naum Papanicolau who throughout the years of my research has proven to be a valuable resource and friend. 


\section{Contents}

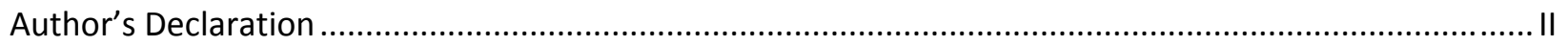

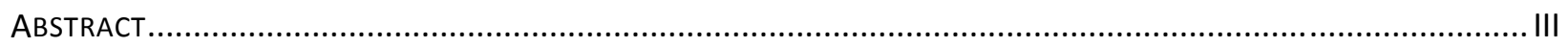

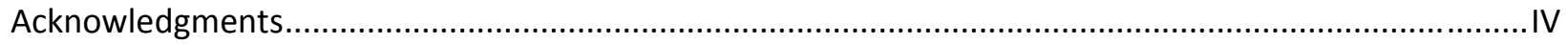

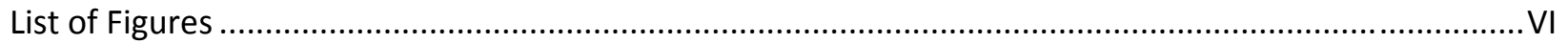

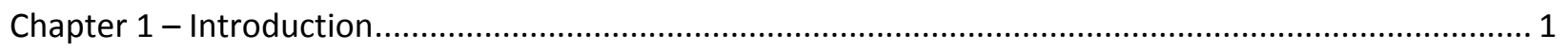

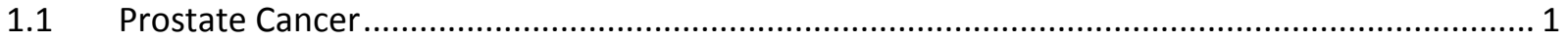

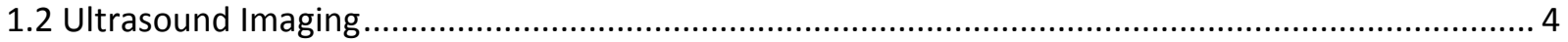

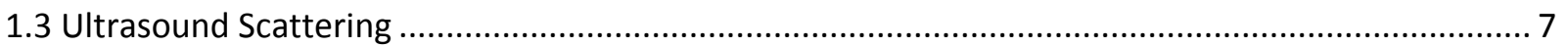

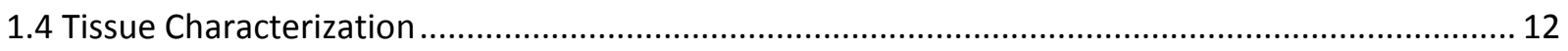

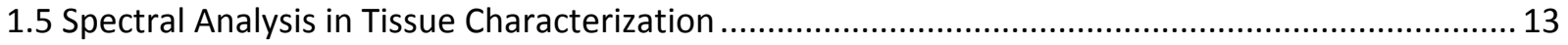

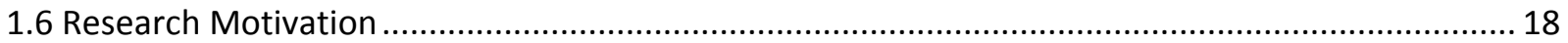

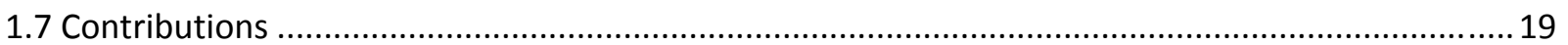

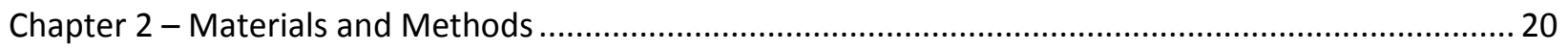

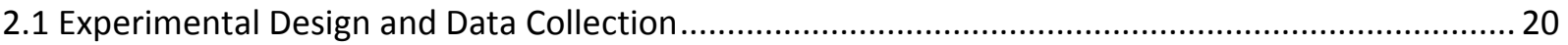

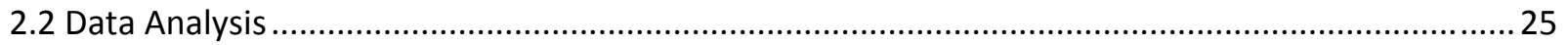

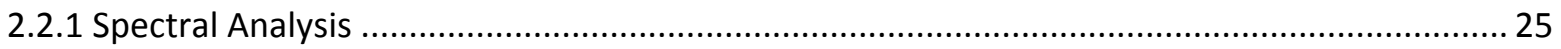

2.2.2 Parametric Image Generation and Analysis..................................................................... 28

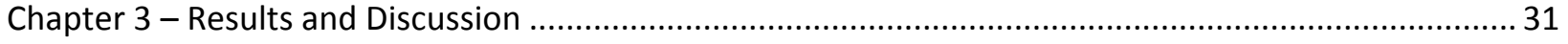

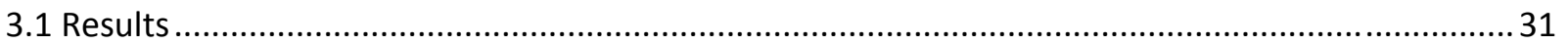

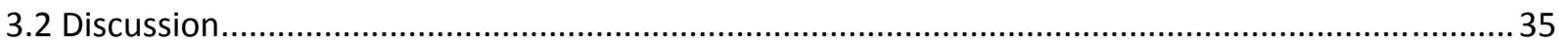

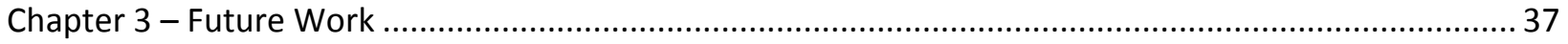

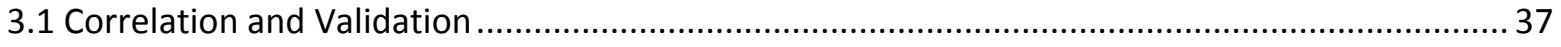

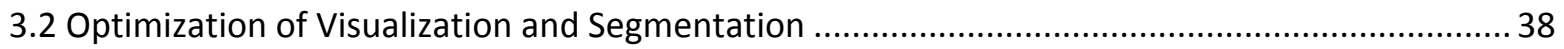

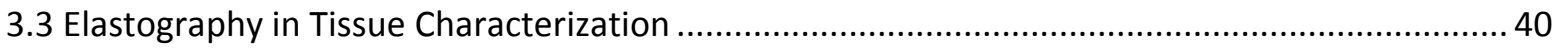

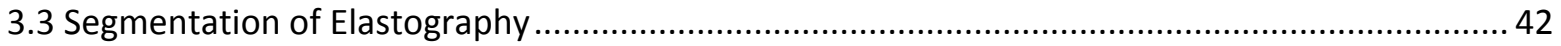

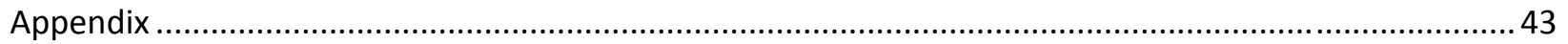

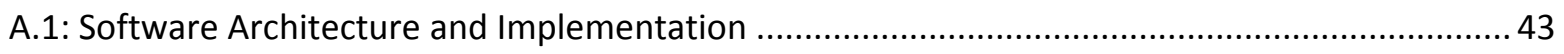

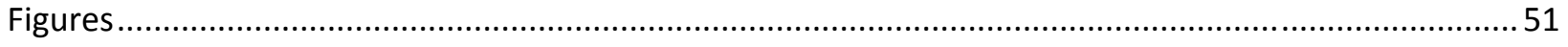

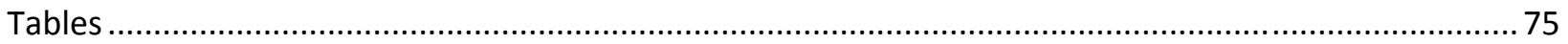

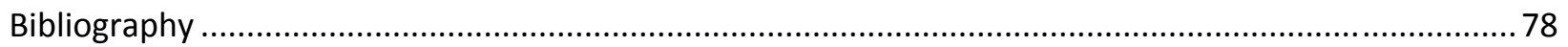




\section{List of Figures}

1. Sinusoidal waveform representation of a sound wave.

2. Longitudinal representation of a sound wave where the particle motion (rarefaction and compression) is parallel with the direction of propagation (Left). Shear wave representation of a sound wave where the particle motion is perpendicular to the direction of wave propagation (Right).

3. Pictorial representation of some of the basic building blocks for an ultrasound transducer. The electrodes apply alternating potential difference causing a piezoelectric effect. As a result the piezoelectric crystal vibrates at a high rate therefore converting the electrical energy into a mechanical sound wave. The result of the electrical input is a corresponding ultrasound pulse.

4. Representative A-mode trace prior to envelope detection through the middle of the prostate acquired using an Ultrasonix RP scanner with a transrectal probe. A typical transverse B-mode image of the prostate composed of multiple A-mode traces after signal envelope detection and processing to create a quality contrast two-dimensional image of the prostate gland (Right).

5. The interaction of the incident plane wave of wavelength smaller than the object which causes portion of the incident wave to reflect and refract. The ratio of the incident wave to the reflected wave requires knowing the acoustic impedance for both mediums $Z_{1}$ and $Z_{2}$ and is dependent on the incident angle $\theta_{1}$ and refraction angle $\theta_{2}$.

6. Values for the mean and standard deviation for spectral intercept and midband fit. These values represent the data from the learning set used in training a neural classifier. The cancerous set was comprised of 29 independent ROI's. The BPH and benign set comprises of a combined set of 60 independent ROI's. (Fellepa at al, Typing of Prostate Tissue by Ultrasonic Spectral Analysis, 1996)

7. (Top) High-frequency ultrasound images of Acute Myeloid Leukemia (AML) cell pellets submerged into saline buffer then treated with chemotherapeutic agent cisplatinum for $0,6,12,24$ and 48 hours inducing a variable degree of apoptotic cell death. (Bottom) Representative corresponding histological analysis stained with H\&E, depicting the degree of 
apoptotic cells present in the pallet.(Czarnota et al, Ultrasound imaging of apoptosis: highresolution non-invasive monitoring of programmed cell death in vitro, in situ and in vivo,1999)

8. (First row) Representative low-frequency ultrasound images of PC3 tumors indicating variability in echogenisity depending on treatment regimen. In left to right order: untreated tumor, $8 \mathrm{~Gy}$ radiation, anti-vasculature microbubble treatment and $8 \mathrm{~Gy}$ radiation followed by, antivasculature microbubble treatment. (Second row) Normalized power spectra taken before treatment and 24 hours following treatment for each group. (Third row) Low magnification light microscopy images of TUNEL stained PC3 tumor slices. (Forth row) Light microscopy images of H\&E stained PC3 tumor slices at high magnification. (Papanicolau, Conventional frequency ultrasound detection of tumor response in vivo to cancer treatment administration,2009)

9. Schematic of the bi-planar transrectal probed used in this experiment. (Courtesy of Vermon S.A.) 10. Schematic of the direction of the scan and the probes realive position to the prostate gland.

11. Depiction of $6 \mathrm{~dB}$ analysis window determined from the power spectra of the reference pulse.

12. The red curve is a representative power spectra corresponding to an ROI representative of the entire prostate grand. The black curve is a power spectra collected from a phantom to be used for normalization, taken with the same machine settings as the one collected from tissue. The blue curve is the resulting normalized power spectra obtained from the subtraction of the tissue power spectra by the phantom power spectra. The linear regression is shown with the yellow line corresponding to the $6 \mathrm{~dB}$ analysis window.

13. The main steps followed in our analysis mechanism are illustrated here starting with the B-mode reconstruction from raw RF data shown on the left most of the image. The second step is the generation of the parametric images for each of the three spectral parameters under investigation. The second image is a representative parametric image generated using the midband fit parameter. The second last image is the corresponding image of the manual segmentation of the parametric image in which only the areas of the prostatic gland are included. Also in this stage the threshold value is discovered and in turn applied to all the parametric slides for each prostate. In the last phase only the thresholded areas where the MBF parameter suggests high likelihood of carcinoma presence in the prostate are extracted. The areas within the bounds of the prostate still present in the image are then added to compute the total are of suspicion.

14. Top row displays ultrasound images reconstructed from RF data. Each individual image represents a stationary plane through the prostate in the direction base to apex. All the images shown are obtained from one representative patient and are separated by a $5 \mathrm{~mm}$ gap in 
between. The second row displays the resulting parametric images of the MBF parameter from the same planes obtained as a result of spectral analysis. The third row displays the resulting parametric images of the spectral intercept obtained from the same planes. The last row displays the corresponding slices obtained from histological analysis. The histology slice are representative $4 \mu \mathrm{m}$ slices obtained from $5 \mathrm{~mm}$ blocks which correspond to the same regions for which the ultrasound RF data was collected. On the histology images areas of prostatic carcinoma are contoured with green color. Areas of abnormal glandular activity are contoured in orange.

15. A graphical representation of the MBF percent area versus the corresponding percent area from the histopathological findings for all the steps collected from Patient 1 (Left). A linear regression analysis is showing with the solid line. The $0-\mathrm{MHz}$ spectral intercept versus the hisptopathological analysis corresponding to the percent disease area as well as a linear regression noted with a solid line (Right).

16. Cumulative scatter plot depicting the percent disease area obtained from MBF parameter versus the percent disease area obtained from histological analysis. The data shown in this graph represents all the data pairs from individual steps collected for the entire patient population which participated in the study. The solid line represents the best fit line obtained from liner regression with the corresponding 95\% confidence interval is depicted with a broken line.

17. Cumulative scatter plot depicting the percent disease area obtained from the spectral intercept parameter versus the percent disease area obtained from histological analysis. The data shown in this graph represents all the data pairs from individual steps collected for the entire patient population which participated in the study. The solid line represents the best fit line obtained from linear regression with the corresponding 95\% confidence interval depicted with a broken line.

18. In this graph the each point represents a patient who participated in or study. The $Y$-axis shows the average percent disease area computed from all the slides belonging to the patient. The Xaxis is the average percent disease area calculated by adding the results of histopathological analysis of each slide pertaining to the patient. The solid line represents the linear regression goodness of fit with the $95 \%$ confidence interval shown in the broken line.

19. In this graph the each point represents a patient who participated in or study. The Y-axis shows the average $0-\mathrm{MHz}$ intercept percent disease area computed from all the slides belonging to the patient. The $\mathrm{X}$-axis is the average percent disease area calculated by adding the results of 
histopathological analysis of each slide pertaining to the patient. The solid line represents the linear regression goodness of fit with the $95 \%$ confidence interval shown in the broken line.

20. High level conceptual model of application architecture depicting the various layers and their scope. The vtkDotNetWrapper acts a middleware layer of communication joining the native .NET framework with the VTK win32 librariers.

21. Representative elastrogram obtained from the same patient shown in Figure 14. The elastogram was obtained using Ultrasonix RP ultrasound system with a curvilinear transrectal transducer pulsed at $10 \mathrm{MHz}$ with a sampling frequency of $20 \mathrm{MHz}$. The color bar on the right hand side shows the color distribution corresponding to the relative tissue stiffness distribution.

22. A visual representation of the various components comprising of the Spectral Analysis Module. Each individual component communicates with the rest through a system of delegates passing through the Main Container.

23. An illustration of the communication pathways between the various components in the Spectral Analysis Module. In this scenario, the System Control invokes a frame change event ( the frame number from an multi-frame ultrasound file) with the Main Control. Since, all the available components are registered with the Main Control; the Main invokes a global change frame event.

24. Visual representation of the display pipeline utilized by the Display Control component. The visualization pipeline above the dashed line shows the VTK objects utilized in the visualization process prior to rendering. The red arrows delineate the classes which define how the dataset will be visualized. These objects can encompass various filters and functions which define the visualization properties of an object and may include color, opacity, lighting, texture, etc. The path shown with black arrows shows the path which the dataset takes from the basic structure through the various filters which change the state of the data to create the volume prior to rendering. Bellow the dashed line is the graphical pipeline. Once the volume object is created and the visualization properties are added, the volume object undergoes through the rendering process. Finally the result of the rendering process is displayed by the rendering window.

25. An illustration of the steps taken in generating parametric images from spectral parameters using a sliding window algorithm. Step 1 defines the size of the sliding window, referring to the number of scan lines ( $x$ direction) and number of data points per scan line (y direction). The window is used to compute the spectral values pertaining to the position at the center of the window. The second step is the extraction of the data point required to compute the averaged power spectra for the window in the current position. The third step involves the normalization 
of the averaged power spectra and computation of the values for each individual spectral parameter, $\mathrm{MBF}, 0-\mathrm{MHz}$ intercept and spectral slope. The values are placed in separate data arrays and the window changes position by one scan line in the $X$ direction. Once the end of the scan lanes has been reached, the sliding window changes position back to the first scan line and window size down in the $Y$ direction. Once the sliding window has traversed the entire image, the resulting parametric images are displayed for the user to apply custom color transfer functions. When the process is performed on an ultrasound volume, the sliding window repeats the process over to the next available frame 


\section{Chapter 1 - Introduction}

\subsection{Prostate Cancer}

Prostate cancer is among the leading cancer related deaths in men, with a high number of newly diagnosed cases yearly. The United States of America National Cancer Institute reported 192,280 new cases prostate cancer diagnosis in 2009 , the same year 27,360 patient deaths were reported as a result of prostate cancer [1]. Prostate cancer, most frequently adeno-carcinoma of the prostate with a tumor growth rate varying from slow to moderate, is generally considered to respond successfully to available treatments. Current acceptable treatments vary based upon the stage of disease and the overall health of the patient. Radical prostatectomy is a surgical method reserved for stage I and II disease where the patient is in good overall health and the tumor is localized in the prostate gland $[2,3]$. Post-surgery, during a typical course of a patient's treatment the institution providing the treatment performs a pathological assessment and diagnosis of the extracted tissue. The main goal for this assessment from a clinical care provider's perspective is to evaluate the margins of the prostate. In this procedure, the tissue is evaluated under high magnification light microscopy in order to determine the proximity of the disease to the boundaries of the prostatic gland. The findings of pathological assessment yield negative margins when there is no disease present at the boundaries of the prostate. Positive margins are an indication that the borders of the prostatic gland have tumour presence which in turn translates to a high chance of the disease having spread outside of the prostatic gland. Since pathological assessment is the current standard in patient care we adopted a similar but more extensive method to evaluate the performance of the spectral parameters. Patients with clinically confirmed cancer present in the prostate gland and/or surrounding tissue ( stages I, II) or patients with stage III disease (invasion of deeper tissue), generally undergo definitive external-beam radiation therapy $[1,4]$. Other treatment modalities being practiced and investigated in clinical settings include interstitial brachytherapy, 
hormonal treatment, cryosurgery, postoperative radiation delivery and cytotoxic agents [1]. The average age, at clinical diagnosis for prostate cancer in men is 72 years of age, but it can be much earlier with PSA testing. Treatment options are largely influenced by age and the general health of the patient as coexisting medical problems can make certain treatments unavailable [1]. It is a common belief that early detection of the disease can improve treatment options as well as long term survival rate of patients.

Screening of patients for prostate cancer is a very controversial topic; studies have shown mixed results and lack of direct benefits with respect to prostate cancer mortality rates of screened patients [5]. A well-known screening mechanism available to clinicians has been digital rectal examination (DRE). A study performed in Sweden attempting to evaluate the benefits of DRE screening which included 9026 men between the ages of 50 to 69 found no difference in the mortality rate between screened patients and control unscreened patients. The 1494 patients who were screened with DRE in a 3 year interval revealed a prostate cancer related mortality rate of $1.3 \%$ (20 patients). The same $1.3 \%$ mortality rate was also observed in the control group of 7532 with 97 cancer related deaths [6]. In the late 1980s prostate specific antigen (PSA) assays became widely available for screening purposes. Prostate specific antigen based testing has proven to add additional value in increasing the number of early detections of prostate tumor. This method is relatively cost effective and reproducible and has added a more objective set of criteria to the screening process. Even though it has been shown that there is no PSA level which is necessary cancer-free, the value of $4.0 \mathrm{ng} / \mathrm{ml}$ is often used as a cut-off for further examination and biopsy [7]. A 10 year case study reported that $71 \%$ of patients with PSA levels above $4.0 \mathrm{ng} / \mathrm{ml}$ had clinically significant cancer in a 5 year span with $91 \%$ specificity over 10 years [8]. It is believed that lowering the PSA level cut-off will increase the number of prostate cancer detections, however it will also increase the risk of false positive patients being subjected to biopsy procedure, which is both expensive and could cause further complications in a patient's overall health [9]. It has 
been demonstrated that in $65-83 \%$ of men with high PSA levels, during the course of 1 year the PSA levels to return back to normal [10-12]. Several causes have been identified as reasons for men to have elevated PSA levels, some of which are biological in their nature and others pharmaceutically induced [13].

Imaging of the prostate gland for the purpose of screening and diagnosis is feasible using various imaging modalities such as magnetic resonance imaging (MRI), computed tomography (CT) and ultrasound. These imaging modalities are characterized by their merits and disadvantages. Even though they all possess the capability of successfully imaging the prostate, transrectal ultrasound (TRUS) is the most commonly used method. The reason for this is attributed to the relatively low cost of modern ultrasound systems in combination with high portability and safety. Unlike MRI and CT, TRUS employs no contrast agent or ionizing radiation, respectively. In terms of ultrasound it does not induce any significant bio-thermal effects when used at clinically acceptable settings [14, 15]. The use of TRUS as an evaluation tool for patients with prostatic carcinoma has demonstrated to have a sensitivity range from $71 \%$ to $92 \%$ for carcinoma of the prostate and $60 \%$ to $85 \%$ for subclinical tumors. Even though TRUS indicates promising results in its ability to detect prostatic disease, it is generally used as a diagnostic follow-up technique rather than an initial screening modality. Trans-rectal ultrasound is the primary imaging modality widely used in contemporary prostate biopsies. There is evidence to suggest that TRUS guided biopsies have ultimately increased the detection capabilities of biopsies by targeting areas with a high likelihood of disease presence $[16,17]$. Considering the vital role that TRUS imaging plays in clinical care, investigating its full capabilities in combination with advancements in signal processing and analysis, can potentially add additional benefits to screening and monitoring mechanism that are currently in place for prostate cancer patients. This work investigates the use of TRUS in clinical settings in combination with spectral analysis methods as a detection and treatment monitoring modality. 


\subsection{Ultrasound Imaging}

Acoustic waves are a form of mechanical pressure transporting energy as they travel through a conducting medium creating alternating particle compression and rarefaction areas. Sound wave propagation can be described as a sinusoidal waveform with a characteristic pressure (p), wavelength $(\lambda)$, frequency (f) and velocity. Figure 1 shows a typical sinusoidal waveform representation of a sound wave with some of its defining characteristics. When acoustic waves travel in parallel to a particle's motion they are called longitudinal waves. This type of motion is observed predominately in fluids. In solids there are two types of wave propagation, longitudinal, and shear waves, the latter where the direction of the wave is perpendicular to a particle's motion. Figure 2 depicts such particle movement in relation to direction of the propagation. Ultrasound is a form of an acoustic wave where the frequency is greater than $20 \mathrm{kHz}$, the upper limit of human hearing. In modern ultrasound imaging systems the ultrasound wave is generated when an electric current is applied to the piezoelectric crystals comprising the ultrasound transducer array. The principle of piezoelectric effect, which has allowed for such technology to be developed, was first discovered by the Currie brothers when they observed that by exerting mechanical pressure to certain crystals an electric potential would be created. The reciprocal of that behavior was proven in 1881 by physicist Gabriel Lippman who, using thermodynamic principles proved that a mechanical deformation can be created when applying a voltage differential to piezoelectric crystals. Figure 3 is a representation of a typical single element transducer. The electrodes placed at the sides of the piezoelectric crystal create an alternating potential difference at a resonant frequency which in turn due to the properties of the crystal causes the crystals to vibrate at the selected frequency. This mechanism converts electrical energy to mechanical sound waves, and since the process is done at very high frequencies the output is an ultrasound pulse. The reverse occurs when the ultrasound wave reflects back from the object under investigation. The mechanical wave front hits the transducer's piezoelectric crystal which in turn then vibrates creating a potential difference. The 
amplitude of the voltage in the created current can be considered equivalent to the pressure amplitude of the ultrasound wave.

Ultrasound transducers employ a "pulse-echo" technique where the electrical signal stimulating the piezoelectric crystal is not continuously on. This allows the ultrasound transducer to first emit an ultrasound pulse then receive the reflection of the pulse which is called the echo. By calculating the time it takes for the pulse to reach the target and for the echo to return to the source the distance between the source and the target can be estimated assuming a set velocity of the ultrasound wave through the medium. When plotting the pressure amplitude estimated by the voltage values versus time, the distance to the object can be estimated. This is typically called an A-mode scan. Since an A-mode scan can only measure distance in one-dimensional space, this method alone cannot be used for imaging. This technique nevertheless was first used successfully by the military in SONAR (sound navigation and ranging) technology where in a pulse-echo manner underwater ultrasound waves were used to locate and range the distance of submerged objects such as submarines. When combining multiple A-mode traces, either by doing a sweep of the object with a single element transducer or by combining multiple elements into an array, a two-dimensional trace of the object can be created and an image formed. By taking the signal envelope of each individual A-mode trace and assigning brightness values to the corresponding intensities of the reflected echo signal, information regarding the reflection properties of the propagating medium can be displayed as an image commonly using a gray scale. Given that the Bmode image is constructed from multiple A-mode traces often the vertical axis in a B-mode image represents the depth and the horizontal axis the width of the investigating object. The gray value of each pixel in the resulting image represents the power of the reflected signal at that position in space. Figure 4 presents a representative two-dimensional transverse image of the prostatic gland acquired using an Ultrasonix RP system with a transrectal ultrasound curvilinear array transducer on the right and on the left a representative A-mode trace through the center of the prostate at the position indicated by 
the vertical dashed line. Visually one can correlate the "spikes" in amplitude in the A-mode trace to areas of higher brightness at the corresponding position in the B-mode image. 


\subsection{Ultrasound Scattering}

Ultrasound imaging captures interactions of an ultrasound wave with the propagating medium (often tissue). Physical properties of the propagating medium can have significant effects on the backscatter signal and the resulting obtained image. The acoustic properties of the material are significant in ultrasound imaging, since images are constructed from the echo signal reflected from the medium. The reflection is a result of an acoustic impedance difference between the materials within the medium. The acoustic impedance of a material is a property defined as the product of the materials density and the speed of sound within the medium; with units of measure are Rayls $\left(\mathrm{kg} / \mathrm{m}^{2} / \mathrm{s}\right)$. Acoustic impedance is a characteristic property of the material and does not depend on the frequency of the sound pulse [15]. The equation for acoustic impedance is given by:

$$
Z=\rho c
$$

where $Z$ is the acoustic impedance, $\rho$ is the density of the material and $c$ is the speed of sound in the material. The speed of sound also can vary based on the composition of the material; thus speed of sound can be derived from the following equation:

$$
c=\sqrt{\frac{B}{\rho}}
$$

where $c$ is the speed of sound in $\mathrm{m} / \mathrm{s}, B$ is the bulk modulus describing the compressibility of the material (the change in pressure that is required to decrease the volume of the material, measured in Pascals), and $\rho$ the material density in $\mathrm{kg} / \mathrm{m}^{3}$.

There is a direct relationship between the wavelength of the incident ultrasound pulse and the source causing the backscatter. This relationship can be grouped into 3 types of interactions: specular, diffuse and diffractive scattering. In general the intensity of reflected sound wave is a function of the 
cross section of the scatter and the intensity reflection ratio (RR) of the scatter. Figure 5 is an illustration of the interaction of a planar incident wave with mediums of varying acoustic impedance. When the cross section of the medium (homogeneous) is greater in order of magnitude compared to the wavelength then reflection and refraction occur. In this scenario when the wavelength of the incident wave is very small compared to the size of the scatterer, the reflection process can be approximated by rays incident to a flat surface where the scattered wave front is a replica of the shape of the scattering object. The associated reflection ratio is a result of acoustic impedance mismatch between the coupling and the scatter medium. The reflection ratio is described by:

$$
R R=\frac{\left(Z_{2}-Z_{1}\right)}{\left(Z_{2}+Z_{1}\right)}
$$

$R R$ is the reflection ratio and $Z_{1}$ and $Z_{2}$ which are the acoustic impedances of the propagating medium and the scattering body respectively. This relation is independent of the insonifying frequency.

Tissue however is comprised of small heterogeneous structures with asymmetric surfaces. The employed ultrasound beam that interacts with tissue is scattered in many directions by small subwavelength point scatterers. This type of interaction is considered to be independent of the incident wave and it is termed diffuse scattering and the tissue scatterers are termed Rayleigh scatters. The portion of the wave that is scattered back to the source is known as the backscatter echo signal. The intensity of the scattered wave under such conditions varies by the fourth power of frequency as was discovered to by Lord Rayleigh [15]. Lord Rayleigh derived the ratio between the intensity of the incident wave and the scattered intensity from rigid spheres of a diameter much smaller than the wavelength as follows: 


$$
\frac{I_{s}}{I_{i}}=\frac{k^{4} a^{6}}{9 r^{2}}\left[\frac{3\left(1-\rho_{2} / \rho_{1}\right) \cos \theta}{1+2 \rho_{2} / \rho_{1}}+\left(1-\frac{\kappa_{1}}{\kappa_{2}}\right)\right]^{2}
$$

where $I_{S}$ is the scattering intensity, $I_{i}$ the incident intensity and the subscripts 1,2 indicating the properties of the scattering object and that of the surrounding medium respectively. Density is denoted by $\rho$ and $\kappa$ is the compressibility, $a$ is the radius of a scatterer sphere, $r$ the scatter distance from the source, $\theta$ is the relative angle to the incident wave and $\mathrm{k}$ is the wave number, which is described by:

$$
k=\frac{2 \pi}{\lambda}
$$

Compressibility $\kappa$ is given by:

$$
\kappa=\frac{1}{\rho c^{2}}
$$

where $\lambda$ is the wavelength, $\rho$ is the density, and $c$ is the speed of sound. In typical clinical frequencies ( $<15 \mathrm{MHz}$ ), the scattering interactions can be approximated as Rayleigh scatters because the wavelength of the ultrasound pulse is orders of magnitude larger when compared with the microstructural tissue scatterer sizes which are on the order of tens of microns. As a result the backscatter signal intensity is dependent on the fourth power of the incident frequency and the sixth power of the scatter's diameter [15].

The third type of scattering occurs when an isonifying wavelength is proportional to the size of the scattering. This diffracting scattering scenario was described for sound by Faran in 1951 and is 
known in optics as Mie-type scattering $[15,18,19]$. The solution for the scattering caused by elastic spheres in water was derived by Faran as:

$$
R=\sum_{n=0}^{\infty} d_{n}\left(U_{n}-i Y_{n}\right)\left(\frac{2 \pi f}{c}\right) P_{n}(\cos \theta)
$$

where $J_{n}$ and $Y_{n}$ are Bessel spherical functions of first and second order, $\theta$ is the relative angle to the incident wave which is equal to $\pi$ for backscatter, $P_{n}$ is the nth order of Legendre polynomial and $d_{n}$ is the coefficient defined by the boundary conditions between the sphere and the surrounding medium.

Another important factor that can affect the intensity of the backscattered signal is attenuation. Attenuation can be defined as continuous loss of mechanical energy as the ultrasound pressure wave propagates through the tissue. The predominant cause for the loss in pressure amplitude is absorption in which the incoming energy is converted to thermal energy. Attenuation is a function of the pulse or sound frequency, the penetration depth and the attenuation coefficient of the medium and is defined as follows:

$$
A=a d f
$$

where $A$ is the attenuation, $a$ is the attenuation coefficient of the medium given in $(\mathrm{dB} / \mathrm{MHz} * \mathrm{~cm}), d$ the distance traveled through the medium, $f$ the frequency of the ultrasound pulse. Because attenuation is frequency dependent, the penetration depth which can be imaged is also dependent upon the frequency employed within a medium with the same attenuation coefficient.

In general the intensity of the backscatter signal is a complex scattering interaction affected by the physical properties of the scattering source such as the effective size of the scatterer, concentration 
of the scatterers in the medium, their density and compressibility and physical distribution in the medium. Given the complex interactions between the ultrasound pulse and the physical properties of the effective scatterers in tissue, a 2-dimentional intensity map (B-mode) is not sufficient to describe the characteristics of the underlying medium, and thus more sophisticated quantitative techniques are necessary to gain more detailed information regarding the underlying characteristics of the microstructure. 


\subsection{Tissue Characterization}

Since a conventional B-mode ultrasound image is reconstructed by taking the signal envelope of the backscatter radiofrequency (RF) signal, which then is processed by various filters to increase signal and decrease noise, a significant amount of information generated by the scattering medium is lost. BMode images can also vary based on the ultrasound machine being used and user specified settings, making it difficult to achieve unified objective interpretations or draw definitive clinically relevant conclusions in the absence of standardization. Ultrasound tissue characterization utilizes various analysis techniques of the backscattered ultrasound signal in order to generate quantitative measurements regarding the underlying tissue micro-structure. Quantitative ultrasound (QUS) techniques employ various methods of analyzing the backscattered radiofrequency signal to interpret various acoustic properties and relate them to features of the tissue microstructure. One such technique is known as spectral analysis which evaluates parameters derived from the frequency distribution of the backscattered signal. This technique has been demonstrated to possess the capacity to differentiate between various tissue types [20]. Statistical methods can also be used to analyze the amplitude distribution of the backscattered signal envelope. Additionally new emerging tissue characterization quantitative algorithms have been developed and used to utilize the ultrasound backscatter signal to measure the elastic properties of tissues in order to distinguish tissue structures. 


\subsection{Spectral Analysis in Tissue Characterization}

Power spectrum analysis is a quantitative ultrasound method that can be used to investigate the scatterer properties within tissue when interacting with an ultrasound pulse. The foundation for quantitative ultrasound relies on converting the echo signals of the transducer from the time domain to the frequency domain, (often by applying a Fourier transform) and interpreting the frequency distribution of the backscattered signal which normally is not utilized during B-mode image formation. Ultrasound spectral analysis has been used successfully as a framework capable of distinguishing tissue types largely through the work of investigators such as Lizzi and Fellepa etc $[20,21]$. A theoretical framework using spectral analysis was established by Lizzi et al in which he demonstrated use of this analytical analysis model of echo signals relating them to tissue acoustic properties [22] . In several studies performed by this group, the use of tissue characteristics such as scatter size, concentration and acoustic impedance where compared with clinical data and theoretical models [23-26]. It was demonstrated that the analysis of the frequency distribution of the backscatter echo signal can provide complementary information in distinguishing tissue types of the retina, liver, lymph nodes and prostate $[24,26-29]$. The analytical model put forth by Lizzi is a three part process. In his method RF data are acquired and the power spectrum is calculated, the power spectrum is then normalized to remove any system transfer functions and parameters from linear regression of the normalized power spectra (or a fitted model) are obtained and related to known tissue features. The power spectra in this thesis were computed as the magnitude of the Fourier Transform values squared for each individual RF scan line (intensity values). In the case of a broadband linear array transducer the power spectra can be averaged across scan lines. Calibration of the spectrum is performed to remove changes in the shape of the power spectrum curve added by the transfer function of electromechanical characteristics of the transducer and other associated electronic components in the acquisition system. A planar reflector placed perpendicular to the ultrasound beam at the center of focal area often a glass plate or metal sheet 
(strong reflectors) (or a tissue mimicking phantom), can be utilized to obtain power spectra for calibration. In this method, Lizzi's group divides the power spectrum obtained from tissue by the one obtained from a strong reflector. The normalization process ensures that the shape of the remaining normalized power spectrum best fits the result of the ultrasound beam with the tissue. Normalized power spectra are often expressed in decibels relative to the calibration curve $(\mathrm{dBr})$. Lizzi has demonstrated that the amplitude of normalized power spectra can vary according to the type of tissue structure. In the case of diffuse vitreous hemorrhages where Rayleigh scattering has been observed, the amplitude obtained from power spectra is $10 \mathrm{~dB}$ lower then power spectra obtained from asteroid hyalosis. Moreover, by applying a linear regression based best-fit line to the normalized power spectra tissue determining spectral parameters can be extracted: mid-band fit as an indication of the overall level of integrated backscatter, the spectral slope of the bes- fit line on the normalized power spectrum as an indication of the average scatter size and the $0-\mathrm{MHz}$ intercept of the best-fit line as an indicator of the scatter concentration. The mid-band fit parameter is the value obtained from the midpoint of the best-fit line within the analysis window. As an indicator of overall integrated backscatter the mid-band fit parameter is dependent upon scatter shape, size, acoustic impedance, concentration and distribution. The spectral slope is the slope of the resulting linear regression best fit line in the selected $6 \mathrm{~dB}$ bandwidth and is related to the average scatterer size within the tissue. The $0-\mathrm{MHz}$ parameter is obtained by extending the line of best fit outside the analysis bandwidth in order to obtain the amplitude value at the $0-\mathrm{MHz}$ point which has been theoretically correlated by Lizzi to the concentration of scatterers within the propagating medium.

Feleppa has shown encouraging results in determining the range of ultrasonic spectral parameters in histopathologically positive prostate samples in comparison with disease free prostate samples where a decrease of approximately $10 \mathrm{dBr}$ in the mid-band fit and the $0-\mathrm{MHz}$ intercept parameter was indicated [30]. In his work, Feleppa uses neural net classifiers to predict likelihood of 
malignancy in the prostate which is then compared to disease free tissue [31-33]. Figure 6 demonstrates the mean and the standard deviation between the three main types of clinically relevant tissue present in the prostate, computed from independent ROI's corresponding with the biopsy sites [30]. In this figure Fellepa et al present data which make up a neural net learning set including 29 regions with confirmed cancer and combined 60 regions of confirmed BPH and benign tissue [30]. The method used to differentiate between malignant and benign tissue incorporated a database of known biopsy tissue samples, ultrasound RF data collected prior to biopsy, histological results of the biopsy for comparison, as well as patient characteristics such as PSA levels and age among other parameters. Multi-dimensional vectors created from the parameters serve as inputs to the neural net classifier, which was initially trained on a subset of the data in order to establish the proper network weights and the layer depth of the multilayer perceptron. After iterations of the training set, the classifier was then provided the remaining data for verification and comparison with histopathological findings. Receiver operator curves $(\mathrm{ROC})$ were then used to measure the performance of the classifier. An approximate $30 \%$ increase in detection was demonstrated through the use of the multilayer perceptron classifier, with the ROC curve areas of $0.844+/-0.018$ in comparison to the level of suspicion (LOS) method used by urologist with an ROC area of $0.638+/-0.029$ [34].

Different research initiated by Kolios and Czarnota demonstrated that spectral analysis was successfully able to detect morphological changes in cellular structure as a result of cancer therapy administration in cell pellets [19, 35-37]. Exposing cell samples comprised of acute myeloid leukemia (AML) cells to the chemotherapeutic agent cisplatin which induces apoptotic cell death, resulting in cells undergoing canonical apoptotic morphological and structural changes of membrane blebbing, nuclear condensation and fragmentation. High frequency ultrasound (20-30 MHz) was used to collect data at 0 , 24, 48 and 72 hours after cisplatin exposure and revealed an increase in backscatter echogenicity proportional to increase in apoptotic cell death determined through histology [35, 36]. Figure 7 
illustrates representative ultrasound images of $\mathrm{AML}$ cell samples obtained at $25 \mathrm{MHz}$ ultrasound and also high magnification histological images to demonstrate the degree of apoptotic cell death. Increases in signal backscatter were found to show a good correlation to increased levels of apoptotic cell death by comparing the ultrasound signal intensity and features with corresponding immunohistochemistry stained cytological samples. Apoptotic changes in cell morphology corresponded to increases of up to 25 times in ultrasound backscatter signal intensity [35, 36, 38].

The work done by Vlad et al recently, demonstrated the ability of spectral analysis to detect tissue changes at a cellular level in an in vivo mouse model. The use of spectral analysis of highfrequency ultrasound $(15-30 \mathrm{MHz}$ ) was be used as a viable tool to monitor changes in cell viability in mouse and rat models, ranging from detection of ischemic cell death in rat livers to monitoring tumor response to radiation treatment as early as $24 \mathrm{~h}$ post-treatment in mouse head and neck tumor models (FaDu and C666-1) [38-40]. The spectral parameters of mid-bandfit and spectral slope exhibited an increase ranging from 6.5 to $8.2 \mathrm{dBr}$ and 0.77 to $0.90 \mathrm{dBr} / \mathrm{MHz}$, respectively 24 hours after being treated with radiation at 0, 2, 4 and 8 Gray [39]. In extending the use of ultrasound spectral analysis for tissue typing Papanicolau et al have demonstrated that conventional low frequency ultrasound with spectral and statistical analysis of radio-frequency echo signals has the capability to monitor the response of various cancer therapies in SCID mouse models employing a PC3 tumor xenograft [41]. In his work, 32 SCID mice where PC3 tumor xenographs were treated with anti-vascular ultrasound activated microbubble therapy, a single dose of $8 \mathrm{~Gy} 100 \mathrm{Kv}$ X-ray radiation, and a combination of both. The mice were scanned using both high (20-30 MHz) as well as low frequency ultrasound at $10 \mathrm{MHz}$ prior to treatment and then again after 24 hours. The study found good correlation in the changes in spectral parameters between the high and low frequency ultrasound data. The changes in the spectral midband fit, slope and $0-\mathrm{MHz}$ intercept agreed with histopathological findings confirming the presence of gross forms of cell death which include apoptosis, necrosis and mitosis. Figure 8 shows representative low frequency 
ultrasound images from each of the four treatment groups as well as the corresponding spectral curves, histopathology analysis images from TUNEL and H\&E stained tissue samples. Additionally the use of quantitative ultrasound methods has been successfully used by Oelze et al to distinguish and characterize between mammary fibroadenomas in rats and mammary carcinomas in mice by investigating estimated average scatterer diameter and acoustic concentration [42-44]. 


\subsection{Research Motivation}

The hypothesis of this thesis is that ultrasound imaging at clinical settings employing a frequency range between $1-10 \mathrm{MHz}$ could be used to quantifiably characterize prostatic carcinoma in patients. The high rate of prostate cancer diagnosis combined with current inefficient methods of imaging and analysis has prompted a great deal of research aiming to improve patient diagnosis and care in the safest and most cost effective methods. As previously stated in the preceding sections quantitative ultrasound methods have been demonstrated to provide a safe mechanism to observe soft tissue structures. It has been demonstrated that spectroscopic analysis of the raw radiofrequency signal has the ability to differentiate malignancy in soft tissues in eye, breast and prostate tissues creating the potential to improve diagnosis. For instance in the case of prostate cancer this should enable clinicians to better target suspicious regions during a patient's biopsy. The use of spectral and statistical analysis methods, both in the high and the low-frequency range possesses the ability to detect changes in tissue micro-structure thus providing the ability to create a mechanism capable of monitoring effects of cancer treatments. The work in this thesis investigates the use of spectral analysis in a clinical setting employing low frequency ultrasound in differentiating prostatic carcinoma in patients and comparing the results with gold standard histopathology and qualitative ultrasound elastography. 


\subsection{Contributions}

The work presented in this thesis was made possible with the collaboration of Dr. Czarnota's laboratory at Sunnybrook Health Sciences Center involving primary experimental setup, patient section and tissue specimen for histological examination. During my involvement in the laboratory I participated in organizing the clinical trial involving 20 patients, performed data acquisition and patient follow-up. My primary focus and individual contribution is in the spectral analysis of the radio-frequency data acquired with emphasis on spectral parameters as presented in this thesis. Additionally I contributed in the development of a visualization software suite to display 3-D parametric mapping of ultrasound images and test this with results of my current work in spectral analysis. The following chapter describes in detail the patient selection, experimental setup as well as the methods used and the results obtained. 


\section{Chapter 2 - Materials and Methods}

\subsection{Experimental Design and Data Collection}

In order to evaluate the viability of using quantitative ultrasound methods to detect and monitor treatment of prostatic carcinoma our experimental approach was composed of the three phases: 1) Data collection, 2) Data analysis and 3) Data verification.

In the data collection phase the data was collected as part of a clinical trial titled "New Conventional Frequency Functional Ultrasound Analysis for Imaging of Prostate Cancer Extent and Response in Human Cancer Patients" at Sunnybrook Health Sciences Centre and approved by the Sunnybrook Research Ethics Board. The trial was designed to include 20 men diagnosed with localized palpable prostate cancer to be treated with radical prostatectomy. The reason for such a criterion was to permit a wide variety of patient PSA levels, age and overall health or external factors. Also it is necessary to mention that the radical prostatectomy treatment method also allowed examination of the prostate tissue specimen after excision for further comparison. Patients with localized prostatic carcinoma were recruited at Sunnybrook Health Science Centre and with their consent; ultrasound imaging was performed prior to surgery.

Table 1 presents the clinically relevant characteristics for the first group of patients that participated in the study. All the patients participating had PSA scores higher than $4.0 \mathrm{ng} / \mathrm{ml}$, with one exception. The participants were diagnosed with $\mathrm{T} 2$ or $\mathrm{T} 3$ clinical stage with confirmed positive biopsies. The ultrasound scans were performed with patients on an operating room table with the patient stabilized in lithotomy position. An in-house rail-based, probe mount fixture was used to mount the transrectal ultrasound probe to the base of the table. The fixture permitted steady gradual calibrated probe movement both in the axial and lateral direction. Scans were performed using an Ultrasonix RP ultrasound system running Sonix RP 3.1 software in investigative digital RF acquisition 
mode. The probe used was a bi-planar transrectal ultrasound probe from Vermon S.A. containing a curvilinear 128 element linear array transducer at the tip of the probe to allow for sagittal RF acquisition. Another 128 element linear array transducer was located beneath the tip of the probe allowing for axial RF capture as shown in Figure 9.

Table 2 presents the acoustic properties of the bi-planar transrectal probe as provided by the manufacturer. During the ultrasound acquisition scan, the probe was mounted to an in-house railing system and inserted into the patient rectal cavity. When capturing transverse RF data the curvilinear transducer was active and the ultrasound beam would in principle be perpendicular to patient's urethra. Using the dials on the railing system, the probe was guided to the base of the prostate which served as the starting position of RF data acquisition. The position of the probe was updated after RF data was acquired by $5 \mathrm{~mm}$ increments following a trajectory from the base of the prostate to the apex, along the rectal wall. The number of steps at which data was collected varied depending on the size of each patient's prostate. Several ultrasound RF frames were captured at each position 20 while the probe was stationary, for averaging of the frames and therefore minimizing slight patient movements during the acquisition period. Figure 10 presents a schematic representation of the probe's directional movement along the rectal wall with transverse single captures representing each stationary step. Along with the raw RF data, acquisition of B-mode data and elastography images took place at each step. The RF data for each step was acquired employing the curvilinear array transducer pulsed at $10 \mathrm{MHz}$. The sampling frequency during the RF acquisition was $40 \mathrm{MHz}$ (chosen to satisfy Nyquist's criteria). While all the other parameters of the ultrasound machine, such as transmit and received power, were maintained constant the only parameter that was varied was the receiver gain. The line density utilized was 512 which with an $80 \%$ sector width permitting for 410 RF lines per frame with an average of 2040 data points per scan line. The exact number of data points varied depending on the depth of the image. The RF data from each scan was saved digitally. In attempting to visualize the 
prostate gland in three dimensions the RF frames from each step were averaged and combined together to create a 3-D representation using linear interpolation to fill the gaps between the steps. Since the stationary RF acquisition steps were $5 \mathrm{~mm}$ apart, a pronounced bias was observed when attempting to visualize the prostate gland in three dimensions due to the lack of sufficient information for the interpolation method used to properly recreate a smooth transitional figure. The lack of more refined increments on the mounting system for the transrectal probe limited the ability to capture RF data at increments smaller than $5 \mathrm{~mm}$. A compromise was made to add one more freehand ultrasound scan in RF acquisition mode in order to encompass the entire prostatic gland in the same direction as the collected steps, from the base of the prostate to apex. An important factor for choosing a freehand sweep is that it does not increase significantly the overall time for which the patient is exposed in a lithotomy position for the duration of the scan. The sweep of the prostatic gland was done ultimately in a freehand fashion which provided more data from a visualization aspect. Approximately 120 frames of RF data were captured during the single freehand RF sweep for an average prostate. However this method does not fully eliminate the error in depicting the prostate in three dimensions, as the scan was acquired freehand and would make the calculation of the probe's position inconsistent due to variability in the speed of hand movements as well as considerable overlap between frames due to the ultrasound beam width and the rate at which data was captured. For this reason the data collected from the sweep was not used in calculations but only as a visual aid.

In a similar manner, the linear array transducer located perpendicular to the curvilinear array on the probe was used to scan in the axial direction. Without extracting the probe from the patient the active transducer was changed to the linear array and the probe oriented in the middle of the prostatic gland, and then oriented to the patient's right most position where the prostate was still visible. Several RF data frames were captured at stationary positions along the middle of the prostate. The increments for each step were $15^{\circ}$ from the patient's right to left. In similar fashion accompanying B-mode data and 
corresponding elastograms were recorded. For visualization purposes a freehand sweep of the prostate was performed using the linear transducer from the patient's right to left. Each patient in the trial underwent ultrasound scans during a single session which lasted approximately 1 hour. This method was relatively non-invasive and considerably minimized the amount of pain and other side effects when compared to current methods of diagnosis such as biopsy.

Shortly after data acquisition (days to 1-2 weeks), patients underwent a radical prostatectomy surgery. The result of a radical prostatectomy procedure is the effective removal of the prostatic gland from the patient, which normally also requires the removal of the seminal vessels. After surgery was performed, the specimen was collected for histopathological investigation. Following the standards of care at Sunnybrook Health Science Centre after surgery the specimen was first prepared by O.R. Pathology where initial an examination of the tissue was done. The specimen was first stained with two dyes on the surface withgreen to mark the patient's left and blue marking patient's right side. Additionally partial tissue was removed for clinical analysis including the seminal vessels; approximately a $5 \mathrm{~mm}$ slice of the bladder neck and a $5 \mathrm{~mm}$ slice of the apex. Following the partial tissue removal from the prostate, the remaining tissue was then sent for whole mount fixation and sectioning. The process of the whole mount fixation used in this study required the specimen to be fixed for 8 up to 24 hours in $5 \%$ formalin. The tissue was then embedded in histomer and mounted in such fashion that the specimen's urethra was horizontal to the surface of the container. Once the histomer solidified, using a Rival meat slicer confirmed $5 \mathrm{~mm}$ slices were sectioned in a serial fashion from apex to base at an approximate $90^{\circ}$ degree orientation, perpendicular to the urethra, also matching the orientation at which ultrasound scans were performed. The slices were then placed in cassettes and submerged again in a formalin solution overnight ensuring that every part of the specimen was fixed. After the fixation process was completed, each slice was then embedded into a paraffin block for processing in a tissue processor. Final sectioning was performed on a Leica 2500 motorized sliding microtome using 
disposable blades. Sections which floated out in at $50^{\circ} \mathrm{C}$ water bath were then placed up on an adhesion- coated $2 \times 3$ inches glass slide. Staining was then performed on each slide, one slide per block. The slide was then stained with haematoxylin and eosin (H\&E) which stains for DNA and nucleic material within tissue. The stained glass slides were subsequently then digitized at maximum resolution using a TissueScope 4000 digital microscope. The digital images were then sent for pathological assessment to a pathologist who in turn examined the section and delineated areas of disease presence and abnormal glandular activity. The results of this process are digital images of the prostate slices with contoured areas of confirmed disease and glandular activity in the same orientation at which the ultrasound scan was performed. 


\subsection{Data Analysis}

After each individual scan was performed, data was saved to a flat file for post processing. The files we exported from the machine allowing for custom built software tools to be used in order to calculate and extract the desired parameters.

\subsubsection{Spectral Analysis}

The RF files obtained during the ultrasound scans contain backscatter intensity data associated with every element and scan line for the particular transducer used. In our analysis of the experimental data we analysed the frequency distribution of the backscatter signal to obtain a further insight of the tissue micro-structure within the prostatic gland. Specifically, we investigated information contained within the power dispersal of the backscatter signal as it is distributed through the frequency range, (power spectrum analysis). A custom tool was developed in our laboratory to run in a Matlab environment to perform spectral calculations. The basic building block of the algorithm was to compute the power spectrum of the RF signal employing the Fast Fourier Transform (FFT) found in the Matlab environment. Matlab made use of the following functions to compute the FFT and its inverse to convert the input matrix from the time domain to the frequency domain and vice-versa.

$$
\begin{aligned}
& X(k)=\sum_{j=1}^{N} x(j) \omega_{N}^{(j-1)(k-1)} \\
& x(j)=\sum_{k=1}^{N} X(k) \omega_{N}^{-(j-1)(k-1)}
\end{aligned}
$$


where $\mathrm{N}$ is the length of the vector and $\omega$ is the $\mathrm{N}^{\text {th }}$ root of unity described as follows;

$$
\omega=e^{(-2 \pi i) / N}
$$

Power spectrum analysis was performed either on the entire RF data matrix or a region of interest which was manually selected. For this reason the equivalent B-mode image was reconstructed from the RF data without performing any filtering or other image enhancing techniques. Reconstructing the B-mode image from the RF data first required taking the signal envelope of the backscatter intensity and passing the resulting values to a log function to logarithmically compress the data for minimal contrast enhancement. The Matlab sequence used was as follows;

$$
Y=10 * \log _{2}(\operatorname{abs}(X))
$$

where $\mathrm{Y}$ is the resulting $\mathrm{B}$-mode matrix and $\mathrm{X}$ is the $\mathrm{RF}$ data matrix.

Once a region of interest (ROI) was selected, the logarithmically compressed B-mode data was disregarded and the corresponding RF data sub-matrix corresponding to the ROI extracted and the power spectrum calculated. In order to perform power spectrum analysis the RF data was converted from the time domain to the frequency domain by applying the FFT of the RF data matrix. The FFT function was applied to every individual RF line in the selected ROI; the resulting magnitude of the computed spectrum was then squared and logarithmically compressed in order to obtain the power spectra in decibel scale. The process was repeated for all individual RF lines present in the ROI and the resulting power spectra vectors corresponding to each individual line averaged across all indices. The resulting power spectrum vector comprises the frequency component and a magnitude component in decibels. In order to remove any system transfer function artefacts the resulting power spectra was 
normalized. Normalization was achieved by calibrating the power spectra obtained from the ROI to the power spectra collected from a reference pulse. The reference pulse used was obtained using the same system settings at which the RF data was collected during clinical data acquisition during the trial. Using the same system settings reference RF data was collected by imaging a tissue mimicking agar embedded glass bead phantom model [45]. After having obtained the RF data from the reference phantom the same process was followed as for the clinical RF data in order to extract a reference power spectrum. Since both power spectra are scaled logarithmically a subtraction was performed to obtain a normalized power spectrum.

$$
\operatorname{NPS}(x)=P S(x)-\operatorname{RPS}(x)
$$

where NPS is the normalized power spectra, PS is the power spectra collected from the patients and RPS is the reference power spectra extracted from the phantom, and $\mathrm{x}$ is the position along the frequency component of the power spectrum vector.

Having obtained a normalized power spectrum, a window of analysis was selected in order to extract spectral parameters related to underlying tissue micro structure. Common practice suggests that the majority of signal power is contained in a $-6 \mathrm{~dB}$ window from the peak magnitude of the center frequency of the transducer. Figure 11 is a graphical representation of the selection of the $6 \mathrm{~dB}$ analysis window from the power spectra of the reference pulse. Having identified the peak magnitude in the reference spectra a $6 \mathrm{~dB}$ window delineates the bandwidth in the normalized power spectrum for which linear regression analysis is performed. A linear regression was applied to the resulting frequency range of the normalized power spectrum using Matlab's polyfit() algorithm implementation. 
A first degree polynomial fit of least squares function was employed as;

$$
p(x)=p_{1} x^{n}+p_{2} x^{n-1}+\cdots+p_{n} x+p_{n+1}
$$

The function finds the coefficients of polynomial $p(x)$ and the result is a row vector of length $n+1$ containing the polynomial coefficients in descending power. Figure 12 depicts the process of normalization of the power spectra. The red curve depicts is a typical power spectrum obtained from prostate tissue using the system previously described. The black curve depicts the power spectrum obtained from the tissue mimicking glass bead phantom as a reference used for normalization. The curve shown in blue is a depiction the normalized power spectra, resulting from the subtraction in a logarithmic scale of the ROI spectrum by the reference spectra, as well as the linear regression applied on the normalized power spectra across the $6 \mathrm{~dB}$ analysis window, shown in yellow.

\subsubsection{Parametric Image Generation and Analysis}

The goal of this study was to investigate the capacity of spectral analysis as a method of characterizing malignancy in the prostate. In order to ascertain this capacity, parametric maps were generated for all three spectral parameters namely mid-band fit, spectral slope and 0-MHz intercept. Employing the spectral analysis method described above the RF data extracted from the ROI served as input matrix to a sliding window algorithm which calculated all three parameters of interest in our study. The size of the sliding window was a user defined parameter. In this study results were obtained using a $4 \times 4$ window from the index being computed thus using $9 \times 9$ data points in order to perform spectral calculations. In this method the result for an input matrix of dimensions $\mathrm{N} \times \mathrm{M}$ is a matrix of ( $\mathrm{N}$ 2 windowsize) $\times(M-2$ windowsize). For each data point in the input matrix, 9 RF scan lines and 9 data points are used to calculate the power spectrum which in turn is normalized, log compressed and the 
corresponding mid-band, spectral slope and $0-\mathrm{MHz}$ intercept values are computed and placed in the corresponding position in the three output matrices over the whole image. Since the selection of the ROIs ensures inclusion of the entire visible prostate captured by the ultrasound data the resulting matrices are parametric representations of the prostate. This permitted the application of a colour transfer function in order to obtain a visual representation of the corresponding spectral parameters. This process was repeated for all the acquisition steps over the prostate for each patient. Once the output matrices were generated for all the desired spectral parameters and the colour transfer function was applied, the data was exported as an image retaining the scale of the output matrix. Using a Java package for bi-directional communication and data exchange from Matlab to ImageJ (MIJ) [46] the parametric images were processed using the tools provided in ImageJ. Once the data was imported into Image the matrix was then normalized to a gray scale with range of 0-255. Having normalized the data to a gray scale the bounds of prostate structure captured at each step was manually contoured. Using the image extraction method in ImageJ the normalized data incorporated within the contoured bounds was extracted from the input matrix in order to eliminate the effect of tissue outside of the prostatic gland in area computations. By extracting the prostate gland region in images, the effective area of the prostate was calculated for each step, and the same computation performed for all the patients depending on the available number of steps per patient.

In order to differentiate between normal prostatic tissue, abnormal glandular tissue (BPH) and prostatic carcinoma, we investigated methods of classifying the values for each of the spectral parameters. Since the range of values for each parameter varied between patients an absolute threshold cutoff to be attributed to a particular tissue type was not used but a histogram threshold value of approximately $67 \%$ of the maximum was arbitrary chosen and evaluated. The set threshold was evaluated on a contoured image, adjusted if necessary to take into consideration patient variability in baseline signal intensity and applied to all images. This was done for each parameter. 
Digital histology images with contoured areas of disease were inputted into ImageJ for quantification. The quantification was performed again in two phases; the first phase computing the total area of disease contoured by a pathologist for each prostate slice. The second phase computed the total area occupied by prostatic tissue. This process allowed for the calculation of the same ratio between disease and healthy tissue. It is important to note, that during the fixation process and whole mount slicing tissue removed from the patient undergoes minor but unknown deformations. This factor had a significant influence in choosing to examine the histology images with respect to determining only a ratio between disease and the remaining tissue. Even though various deformable image coregistration methods using finite elements which could account for such deformations have been proposed in the literature, their scope is not the goal of this thesis. Having established the same ratio in determining the presence of disease in the prostate gland for both the parametric images generated using spectral analysis and the pathological assessment which is currently the gold standard practice in patient care this ratio was employed as the criterion of evaluating and validating our findings.

Since the ultrasound scans were performed at static steps with $5 \mathrm{~mm}$ spacing, and the whole mount histological slicing was performed at the same frequency, a general comparison should be possible between the percent disease areas computed from the spectral parameters and the histological slices. 


\section{Chapter 3 - Results and Discussion}

\subsection{Results}

In this study we have achieved to create a basic framework for using low frequency ultrasound at clinical settings to extrapolate areas of suspected disease in the prostate. Results indicated the ability to identify regions of the prostate with a high likelihood of disease presence. Of the three spectral parameters investigated, the spectral mid-band fit and the spectral $0-\mathrm{MHz}$ intercept showed a considerable degree of sensitivity to changes in the internal structure of the prostate gland. In contrast, spectral slope which is an indication of average scatter size did not seem to be sensitive to the presence of cancer regions in the prostate. Figure 14 is a visual representation of general findings illustrating the various aspects of our analysis. The top row depicts standard B-mode images from one patient reconstructed from the raw RF data without any additional signal altering modifications. Each of the Bmode images represents an independent scan plane through the prostate, separated from a $5 \mathrm{~mm}$ gap between steps. The B-mode images allow for capturing hypo echoic areas, as they are representations of the backscatter intensity from the prostate, however they do not convey very useful information which can be attributed to morphological changes in the tissue microstructure and its interactions with the ultrasound pulse. The second row illustrates the results of spectral analysis, converted into parametric images of the prostate. Each image corresponds to the same spatial step and is obtained from the corresponding B-mode image shown in the first row. The mid-band fit parameter indicated relative variations ranging between 9 to $15 \mathrm{dBr}$ ( $\mathrm{dB}$ relative to the calibration curve) between areas of gross disease presence and areas of normal tissue. Visual analysis demonstrated that data were sensitive enough to permit delineation of bulk disease presence in the prostate. The images in the last row are digitized images from the nominally corresponding hematoxylin and eosin stained representative $4 \mu \mathrm{m}$ thick slices, taken from $5 \mathrm{~mm}$ tissue blocks corresponding to each ultrasound acquisition. The areas of disease in the histology images correlated fairly well with suspected areas of 
disease calculated using the mid-band fit parameter with the resulting parametric images. The method of analysis using the MBF parameter indicated a $9.1 \%$ area of potential disease in the MBF parametric image compared to $7.8 \%$ disease area in the H\&E stained image for the first slice. The reamaining representative slice results were $19.28 \%$ ultrasound to $18.2 \%$ in H\&E (slice 2 ), $15.1 \%$ ultrasound to 12.3\% H\&E (slice 3) and 8.7\% ultrasound to $8.8 \% \mathrm{H} \& \mathrm{E}$ for slice 4 for this representative patient.

In contrast to the MBF parameter, the $0-\mathrm{MHz}$ intercept parameter did not demonstrate a comparable degree of correspondence. Figure 14 - row three displays the corresponding $0-\mathrm{MHz}$ intercept parametric images, calculated at the same steps as the B-mode and parametric MBF parameter for this patient. The corresponding results calculated in the manner discussed above were as follows: $9.7 \%$ disease calculated from the $0-\mathrm{MHz}$ compared to $7.8 \%$ obtained from the histological analysis for the first slice. Moreover, a $6.8 \%$ disease value was calculate compared to $18.2 \%$ (slice 2 ), 5.6 \% ultrasound to $12.3 \% \mathrm{H} \& \mathrm{E}$ (slice 3 ) and $5.9 \%$ ultrasound to $8.8 \% \mathrm{H} \& \mathrm{E}$ for slice 4 . Table 3 contains a summary of the results for the first patient.

A summary of data for the individual prostate specimen was calculated by adding individual frame data and calculating an average percentage area of disease. Similarly, histopathological averages were calculated by determining the total area of demarcated malignancy and averaging over the entire specimen volume. Histopatological results for the first patient resulted in an $11.8 \%$ average area of malignancy, compared to the calculated average percent disease area obtained from the spectral parameters indicating a $13.5 \%$ disease area for MBF parameter and a $7.0 \%$ disease area obtained from the $0-\mathrm{MHz}$ intercept parameter. 
Figure 15 displays data obtained for a single representative patient, summarizing computed parametric average areas. Individual points on the left graph represent the corresponding percent disease area computed for the MBF parameter (left graph) versus the area found in the histological analysis for individual steps along the prostate gland specimen. The graph on the right similarly shows the resulting graph corresponding to the spectral 0- $\mathrm{MHz}$ intercept data. Additionally, a linear regression best fit curve is plotted with a corresponding $95 \%$ confidence interval. The linear regression analysis revealed an $\mathrm{R}$-squared value of 0.894 for the MBF percent disease area but only a R-square value of 0.1196 for the $0-\mathrm{MHz}$ intercept parameter.

Following the same methodology, a summary plot of all data pairs between each individual spectral parameter area calculations and the corresponding histological analysis results for individual steps analysed across all patients was generated. As the analysis method was consistent across all the patients, in order to increase the sample size to permit for a more comprehensive statistical analysis of the patient population, the individual scan steps across all patients were combined into one dataset of corresponding data pairs. Figure 16 provides the corresponding graphical summary of all corresponding parametric and histopathologic data pairs for calculated percent disease areas from the ultrasound data to observed percent disease area determined from pathology for all the patients participating in our study. In order to assess our results a linear regression based goodness of fit test was performed across all data pairs, shown by the solid line along with the corresponding $95 \%$ confidence interval indicated by the broken line. The goodness of fit test revealed an R-square value of 0.8232 across all data pairs. Assuming a non-Gaussian distribution, a non-parametric Pearson correlation test was performed revealing an $r$ value of 0.9073 with a $95 \% \mathrm{Cl}$ of 0.8330 to 0.9494 indicating a significant correlation (alpha $<0.05$ ) with a P-value less than 0.001. 
Figure 17 presents results of data for all patient data pairs for the $0-\mathrm{MHz}$ percent disease area with corresponding percent disease obtained from histopathological analysis across all analyzed data pairs. The goodness of fit test for the $0-\mathrm{MHz}$ intercept parameter revealed a relatively poor $\mathrm{R}$-square value of 0.2775 indicated with a solid line in Figure 17. Performing a non-parametric Pearson correlation test on the spectral intercept parameter in comparison to histology findings revealed a correlation coefficient Pearson $r$ value of 0.5268 with a $95 \%$ confidence interval of 0.2653 to 0.7161 and a P-value of 0.003. Furthermore, in addition to analyzing data on individual slice basis a goodness of fit test based on the average disease area, was computed using the spectral MBF parameter and histological results. Figure 18 displays the graphical representation of averaging the MBF and corresponding histology pairs for each patient individually. The average percent disease area computed from the MBF parameter is shown on the $\mathrm{Y}$-axis, and the corresponding average disease obtained from histopathologic analysis is plotted on the X-axis. A goodness of fit test using linear regression revealed an R-square 0.9885. A nonparametric Pearson correlation test revealed a significant correlation (alpha $=0.05$ ) with an $r$ value of 0.9943 with a $95 \%$ confidence interval 0.9599 to 0.9992 and a two-tailed P-value of 0.0001 . The linear regression line is shown with a solid line in Figure 18 and the $95 \%$ confidence interval with a dotted line.

In contrast, inspecting the averages computed areas using the $0-\mathrm{MHz}$ spectral intercept showed an $\mathrm{R}$ square value of 0.5426 for the linear regression goodness of fit test. The Pearson non-parametric correlation test indicated a non-significant (alpha $\neq 0.05$ ) $r$ value of 0.7366 with a $95 \%$ confidence interval -0.372 to 0.9582 and a two-tailed P-value of 0.059 . Figure 19 shows the corresponding graph of the averaged $0-\mathrm{MHz}$ spectral intercept. 


\subsection{Discussion}

In conclusion we have established a preliminary analysis framework to quantify soft tissue types using quantitative ultrasound spectral analysis to identify potential areas of malignancy in the prostate. From our investigation, we found a significant variance in the range of values for each of the spectral parameters between patients. Variation in the range of the spectral parameters can be attributed to several factors that greatly impact ultrasound imaging such as overall size, density and internal structure of the prostate varying from patient to patient. The fact that all the participating candidates are patients with biopsy confirmed presence of prostatic carcinoma allowed for the patients to be evaluated as distinct target subset. Based on our results the spectral mid-band fit parameter appears to be most sensitive to the presence of prostatic carcinoma formations in the prostate. Comparing the results of estimated percent disease areas computed using the MBF parameter to those provided from histopathological analysis, MBF values were observed to be greater than the current histology gold standard. These observations lead us to believe that the presence of disease is detected with a high sensitivity. Conversely, the spectral $0-\mathrm{MHz}$ intercept does not indicate a comparable sensitivity as the results obtained from the histopathological analysis. The $0-\mathrm{MHz}$ intercept parameter based disease estimations were observed to be lower than those obtained from histology. Furthermore, the spectral slope was observed to be invariant to the presence of prostatic carcinomas. The high variability observed in the spectral slope parameter as an indicator of average scatter sizes suggests that the prostate gland is highly heterogeneous and moreover the size of tumour cells do not appear to demonstrate a consistent variability in comparison to non-malignant prostatic tissue. This may be related to the influence of higher order glandular prostatic intraepithelial neoplasia which changes with carcinoma development, as well as changes occurring at cellular level. The results presented in this thesis are in general agreement with similar type of studies found in the literature. In comparison with work done by Feleppa's group in tissue characterization of the prostate our findings were in general 
agreement. Our results indicated that spectral analysis could be used to detect malignancy in the prostate. This work demonstrated that the mid-band fit parameter is most sensitive to the presence of tumour in the prostate; Feleppa's group has demonstrated similar results with this respect. The midband fit values in the cancerous regions exhibited $\sim 10 \mathrm{~dB}$ average lower than cancer free areas. The 0 $\mathrm{MHz}$ intercept as an independent parameter exhibited a relatively poor performance in identifying the tumour extent in the prostate. In contrast Feleppa's group combines all the spectral parameters with external patient factors such as age and PSA in their classifications of tumour regions. The findings of our study were in complete agreement with Feleppa's groups with respect to spectral slope parameter exhibiting high variability and inconsistency in predicting potential areas of disease. 


\section{Chapter 3 - Future Work}

\subsection{Correlation and Validation}

The goal of the work presented in this thesis was to investigate the use of conventional low frequency ultrasound used in clinical setting as a mechanism to characterize and quantify malignancy in the prostate. It was demonstrated in this thesis that changes in tissue microstructure caused by the presence of prostatic carcinoma can be quantified employing ultrasound at clinically relevant frequencies. The efficacy of the proposed framework, which at its core utilizes spectral analysis, was demonstrated using a subset of patients with biopsy-confirmed presence of prostatic carcinoma. The examination of the power spectra obtained from patients in vivo was indicated that the spectral midband fit parameter and $0-\mathrm{MHz}$ intercept can be utilized to quantify areas of suspected disease in the prostate. The verification of the results was performed by comparing putative "disease" areas detected via the spectral parameters with the current accepted clinical standard, histo-pathological analysis. The work presented in this thesis is a correlation between the data collected using low-frequency ultrasound with histological analysis images obtained from whole-mount histology. The findings of this study demonstrate a relative correlation of the results of spectral analysis compared with histological analysis with the degree of correlation depending on the particular spectral parameter. In order to further improve the correlation between spectral analysis and histological analysis a more precise spatial data acquisition and analysis mechanism is required. In order to improve the results obtained from spectral parameters further investigation into optimization on acquiring the ultrasound data and its analysis is required. Also, new advancements in ultrasound transducer technologies in the development of 2dimensional transducer arrays may provide more accurate results.

Three-dimensional histology is another mechanism which may permit improvements in spatial correlation between quantitative spectral methods and the histological analysis. As previously mentioned the histological analysis was obtained from representative $4 \mu \mathrm{m}$ slices obtained from $5 \mathrm{~mm}$ 
blocks. In addition, due to regulatory reasons which ensure optimum patient care, two sections were removed from the specimen obtained post-surgery. Each section removed was approximately 4-5 mm, and removed from the apex and the base of the prostate respectively. Utilization of 3-D histology can make available multiple representative slices obtained from each individual $5 \mathrm{~mm}$ tissue block. When combining the slices from each block a far more accurate three dimensional histological model of the prostate gland could be recreated. This method may improve spatial correlation between histological analysis and ultrasound imaging which is collected at $5 \mathrm{~mm}$ increments, as it may account for sudden changes in tissue structure in more refined and accurate spatial grid.

\subsection{Optimization of Visualization and Segmentation}

In order to visualize the results obtained via spectral analysis of the backscattered ultrasound signal, a visualization model was implemented. The visualization and analysis framework utilized in this thesis is based on a two dimensional analysis method which is then applied to a stack of ultrasound frames in order to create a three dimensional parametric volume of the prostate for each individual spectral parameter. The analysis framework was implemented using Visual $\mathrm{C}++(\mathrm{VC}++)$ in a Visual Studio environment based on the .NET 2.5 framework. This implementation choice allowed for rapid developing of graphical user interfaces (GUI) while utilizing the execution speed advantage provided by compiled languages into native machine code. While all the spectral calculations of the ultrasound backscattered signal were performed in Visual C++ the visualization was performed entirely using Visualization Tool Kit (VTK) integrated into the .Net framework. VTK is an open source software library which allows for three dimensional visualization of computer graphics as well as powerful modeling and processing of image datasets [47]. The integration of VTK with the .NET platform was accomplished

using as middleware a set of .NET wrapper classes developed by Dr. Václav Skala and Milan Frank $[48,49]$. Figure 20 demonstrates the high level architecture of the main building blocks on which a graphical application is built within the .NET framework as proposed by Skala and Frank. 
The analysis and visualization software created was based in a combination of a structured and event driven architecture comprised of several independent modules. The analysis and data access modules were designed using a monolithic architecture acting as self-contained event driven applications. In contrast the data visualization aspect was designed as filter - pipeline architecture while employing an object oriented approach. The software package was comprised of a spectral analysis, parametric generation, reporting and batch processing, data feed and repository, and a visualization module and a main control user interface. The details of regarding the implementation are provided in Appendix X.

While the software developed is a powerful tool capable of analyzing spectral data for user specified ROls, it lacks the ability to independently identify the structure of the prostate accurately. As a result manual selection of the prostate region is required. The implementation of segmentation algorithms in image processing can significantly improve the accuracy in the selection of ROls for the purpose of accurately calculating the ratio of malignance in the ultrasound images.

Furthermore, improvements in the thresholding methodology are required in order to accurately identify areas of suspected disease in the parametric images generated from the spectral parameters. Currently the thresholding of the parametric images was performed manually using arbitrarily determined guidelines. The manual selection of the threshold cutoff value does not allow for the use of spectral analysis to be utilized on an unknown population sample. Artificial intelligence (A.I.) methods can be employed in the future in order to improve the thresholding mechanism and ultimately the identification of suspected areas of disease in the ultrasound images. The most prevailing implementations of A.I. involve the use of neural networks, more specifically multilayer perceptron classifiers [27, 30]. Support vector machines (SVM) are another type of classifier which have been demonstrated to successfully perform classification of multi-dimensional datasets. SVMs can be utilized 
to consider not only the results of the spectral analysis parameters but also factors external to ultrasound imaging such as PSA levels, age, ethnicity and other clinically relevant patient facts.

\subsection{Elastography in Tissue Characterization}

Elastography presents another method of employing ultrasound waves as a method of quantitative analysis, and does this by exploiting elastic properties of soft tissue. Common practices suggest that cancerous lesions very often are significantly stiffer then the surrounding tissue which enables physicians to use palpation to examine and detect tumors on accessible organs such as breast and prostate [50-52]. However the ability of palpation to detect potential cancerous regions depends greatly on the ability of the professional administering the exam and it has limited depth range. More importantly the results of palpation are not easily quantifiable and cannot provide accurate measurements of size and location. Ultrasound elastography imaging attempts to provide an alternative to palpation by providing a quantifiable visual map of the elastic properties of tissue at an increased penetration depth. Generally, soft tissue methods of imaging can be grouped into two categories: in the first group tissue deformation and strain parameters are extrapolated under quasi-static compression, and in the second category, dynamic compression or natural vibrations are used [52-54]. Both methods estimate tissue deformation by using time delay techniques by tracing the tissue displacement or relaxation prior to and post compression. Based on the imaging method used and the type of processing of the RF echo signal a variety of elastograms can be generated depicting stiffness variability including measures such as axial strain, lateral strain, Young's and Poisons modules [52]. Since the realization of utilizing ultrasound technology to examine mechanical elastic properties of soft tissue several computational methods have been developed such as time-domain cross correlation, power spectral analysis, Fourier base speckle phased tracking and adaptive time-domain cross correlation. Conventionally practiced mechanisms that estimate the local axial strain compute and display the gradient of displacement that the ultrasound speckle pattern exhibits under compression. The time 
domain cross correlation (TDCC) obtains the displacement by interpolating the maximum peak of the correlation function between corresponding RF A-lines before and after axial compression is applied. Time stretching of the RF lines is performed to reduce the noise and improve the accuracy of correlation between pre and post compression $[52,54,55]$. It has been demonstrated that the estimation of the elastic properties of soft tissue can be used to differentiate potential cancer regions in vivo. The use of elastography has been applied in the detection of large breast tumors [56]. Elastography can also be used to both detect and potentially to guide biopsies of the prostate [50]. Preliminary methods suggest the feasibility of obtaining high quality elastography images of the prostate using current transrectal probing techniques. Current brachytherapy practices use a water balloon of known volume around the transrectal probe for coupling reasons. The water volume in the balloon can be changed in a controllable fashion allowing for the pressure to be calculated and therefore a known compression would be applied to the prostate. This method permits creating elastograms with a low contrast to noise ratio using current clinical settings. 


\subsection{Segmentation of Elastography}

The current implementation of elastography image formation in the Ultrasonix RP system used in this study allows for the formation relative elastrograms. The elastograms obtained in the study were created using an adaptive time-domain cross correlation algorithm. In essence, this method compare the individual RF lines from one ultrasound frame to the next creating a cross correlation matrix. The coefficients of the cross correlation matrix are interpreted as a relative scale depicting tissue compressibility as it varies through time. The higher correlation coefficients are interpreted as a result of lesser compressibility which is an indication of harder tissue presence. A representative elastogram collected using the previously mentioned algorithm on an Ultrasonix RP system is shown in Figure 21. The pulsing frequency employed was $10 \mathrm{MHz}$ with a sampling frequency of $20 \mathrm{MHz}$. The stiffer tissue regions are depicted in dark blue color relative to the softer regions depicted in red, the relative color scale is shown on the right hand side of Figure 21 . The cross correlation method of forming elastrograms from ultrasound provides information when used as a ratio between two regions of interests. However, this method lacks quantitative data to classify tissue when the goal of the investigation is to measure and identify areas of a specific stiffness. Since this method compares the relative stiffness between two or more regions it requires that the user must have knowledge of the regions of interest prior to elastrogram collection which then in turn will provide the ability to obtain a relative stiffness ratio between those regions. For the purpose of the study a more absolute stiffness measurement technique is require with a controlled experimental setup. However, with the use of more advanced A.I techniques the relative stiffness values maybe prove to be a valuable addition when combined with spectral analysis methods and other patient characteristics and provide a broader perspective when attempting to classify tissue type in the prostate. 


\section{Appendix}

\section{A.1: Software Architecture and Implementation}

The software package presented in this thesis was created to overcome some of the limitations that exist in the current Matlab environment. Although, Matlab is a powerful computational environment that provides a distinct advantage and significantly simplifies the process of working with complex mathematical problems and matrix manipulations, it lacks in the ability to visualize and render complex scenes comprised of large amount of data. Also, since Matlab is built on Java, program interfaces built in Matlab generally experience a slower performance since the resulting code needs to be interpreted by the Java Virtual Machine (JVM). In order to improve performance the proposed solution makes use of the Graphical Processing Unit (GPU) to perform the visualization tasks using VTK which is built as a graphical library interfacing with OpenGL. Computational performance was optimized by removing the JVM interpretation layer and coding in Visual $\mathrm{C}++$ therefore compiling the code directly to machine language designed for Intel $x 86$ and $x 64$ platforms. The application layer is built on top of DotNet framework 3.5.

A bottom -up software design paradigm with rapid prototyping was used to build the software. Utilizing Object Oriented programing principles the software package was compartmentalized into independent modules. Each individual module was further compartmentalized into six components Control Menu, Data Control, Display Control, System Control, Data Repository and Main Control. Each component is designed as abstraction therefore every module added to the package needs to implement these interfaces. The software was designed to be an event driven architecture and the communication between each component was achieved using a global delegate system. In essence when an event is raised the corresponding delegates are invoked and each component has sufficient capacity to update itself appropriately. The Control Menu functions as the main hub for file 10 
operations such as opening the ultrasound files and saving operations performed on the data. Data Control acts as properties display and interactions component pertaining to each type of analysis performed. System Control is the main component responsible for handling visual user operations performed on the data prior analysis such as selection of ROls, frame changes, frame extraction etc. Data Repository is a component not visible to the user which maintains the state of the data at all time. It contains all the data and external information required to perform all the types of analysis available in each module as well as the results of such analysis. The Data Repository functions as the main hub where each component independently updates the data depending on individual requirements and acts as a temporary storage container. Display Control is the main visualization component which its primary responsibility is to visually represent the ultrasound data and handle visual user interactions with the data. Since all the visualization operations are performed in the Display Control the integration of VTK with each module is done here. The Main Control is the container which initiates execution keeping track of each individual component within a module and updates them accordingly. There are three main modules included into this initial platform, Spectral Analysis Module, Parametric Module and the Reporting Module. Figure 22 is a visual representation of each individual component comprising of the Spectral Analysis Module. The position of each individual component does not change through the various modules. However, the appearance and the enabled functionalities of each component vary.

Since the behaviour of each module is driven either by user events or change in the state of data, the communication process between the main components comprising each module is achieved in a two-step delegate system. When a component raises an event, the invoked event is delegated to the Main Control component, step one. The Main Control upon receiving the invoked event raises a global even to all the controls. Only when a global even has been invoked from the Main Control then each individual component updates itself accordingly. This communication process ensures data integrity at all times without user performance. This architecture allows for scalability of behaviour updates of 
individual components without affecting the others. Also, the addition of new components into the Main Control container can be performed with easy since the delegate interface is the identical even though the behaviour and functionality varies. Figure 23 is shows a demonstration of a of the communication pathways between the components registered with the Main Control of the Spectral Analysis Module during a user invoked change frame event. Upon the user manually changing the selected frame from a multi-frame ultrasound file, the System Control component invokes a delegate with the Main Control. It is the responsibility of the Main Control to invoke a global change frame event with all the registered components of the specific module. Only upon receiving the global delegate invocation of change frame event from the Main Control all the components registered will update accordingly. The component which originated the event does not update itself until the global event is raised from the Main. During the update process components may communicate directly with the Data Repository to retrieve, insert or update existing data.

The ultrasound RF data as well as the parametric images are stored in the Data Repository component. Each individual set of data to be displayed is stored into a vtkImageData object. The class vtkImageData is a basic type of data structure designed specifically to accommodate $2 \mathrm{D}$ and $3 \mathrm{D}$ images. There exist four main properties that define the vtkImageData structure. Dimensions of the image define the size of the image as the number of data point comprising the image or volume. Origin defines the three dimensional position in space of the point $(0,0,0)$. Spacing property defines the voxel dimensions and scalar type defines the primitive data type comprising the image such as integer, float, double etc.

The visualization component of each module is contained within the Display Control component. The display pipeline is a demand driven pipeline because the rendering process occurs as needed. In essence only the parts of the volume visible to the user are rendered, as the view of the user 
changes the rendering process demands the new updated data to be proceed through the pipeline as needed. This type of rendering makes the memory requirements significantly easier to manage by average PCs because it does not require the entire data comprising the volume to be loaded onto the graphics memory. However, this method does require the entire volume data to loaded and kept on the system memory for the duration of the display pipeline.

The display pipeline is divided into two parts the visualization and the graphics pipeline. The visualization pipeline comprises of the volume data source with the corresponding filters and data mappers. The visualization pipeline comprises of three data conversion filters and a custom piecewise mapping. The filters used are the vtkImageMathematics which allows for the extrapolation of the absolute value from RF data set. Second, the output of the absolute value filter serves as input to the vtkImageLogarithmicscale filter converting the data to log scale. Since, the requirements of the mapping filters need the dataset to have a scalar type of unsigned char, the output of vtkImageLogarithmicScale is used as input to the vtkImageCast filter which in turn converts the scalar types from integers to unsigned char. In order to define the vtkProp properties of the volume, two VTK functions are defined. The first defines the opacity of the volume as a piecewise function using the vtkPiecewiseFunction class, allowing for the definition of the degree of transparency as it is distributed along the pixel value range. The color distribution is handled by the vtkColorTransferfunction filter in which the RGB color space and the distribution range of pixel mapping is defined. The opacity and the color transfer function are combined to create a vtkVolumeProperty object. Also, in the vtkVolumeProperty object the interpolation between the voxels is defined, cubic interpolation was used in this implementation. The volume properties are passed to the mapper object along with the data source for the visualization to occur. In this implementation vtkVolumeRayCastMapper was used in combination with the vtkVolumeRayCastCompositeFuntion which defines the type of ray mapping. 
The graphics part of the pipeline starts with the creation of the object to be rendered which defines the 3D volume. The 3D ultrasound dataset outputted from the vtkVolumeRayCastMapper is passed as an input connection a vtkVoume object. The next step in the graphics pipeline is defining a vtkRenderer objects which performs the rendering of the volume. Each individual renderer is capable of rendering multiple graphics objects. The rendering process takes place in a renderer window. The vtkRenderWindow object is capable of accommodating multiple renderers and defines the world in which each individual rendering process takes place. The interactions between the user and the objects in the rendering window are handled via vtkRenderWindowInteractor class objects. Multiple interactors can exist in a rendering window for our purposes only one interactor is associated with the volume object. Figure 24 is a graphical representation of the display pathway that the ultrasound volume dataset undergoes. The pathway is divided into two parts the visualization part transforming the raw data into a volume with the desired visualization properties. The properties that can be defined may include opacity, color, texture, contouring and various lighting and mapping effects. The second part of the pathway encompasses properties that define the rendering process as well as the display scene. Each display scene is comprised of actors to be displayed, rendering processes and other global properties.

The interactor used by the Display Control allows the user to perform simple operations in the scene. These types of operations do not affect the overall processes flow of the application and only include operations such as zooming in and out of the displayed ultrasound volume as well as rotational transformations. The user interactions associated with the selection and visualization of ROls are handled by a combination of VTK widgets and data extraction objects. Although, VTK has at its disposal a variety of widgets, the implementation of the Spectral Analysis Module uses a vtkBoxWidget object. Other widgets such as the spline widget may be utilized at a later time to allow the user the selection of irregular shape ROls. The vtkBoxWidget class allows of the creation of a 3D rectangular box object to 
be placed in the scene along with the ultrasound volume. The advantage of utilizing VTK widget objects is that they provide a comprehensive set of interaction capabilities while being associated with graphical object present in the display scene. The widget class contains its own internal visualization and graphical pipeline without requiring a lot of intervention from a development perspective. In this application the vtkBoxWidget object was utilized in order to draw on the scene a visual wireframe which delineates the volume to be extracted for spectral analysis. The faces of the box widget can be moved freely by the user in order to adjust the volume prior to extraction. The six points defining the box widget in combination with the voxel dimensions of the rendered ultrasound volume were used to compute the dimension for the volume needed to be extracted. The extraction process itself was performed using another VTK class object called vtkExtractVOI. The input connection to the vtkExtractVOI object was the unfiltered and unprocessed dataset as it was first stored in a vtkImageData structure. Once the desired volume was extracted the Display Control inserts the extracted vtkImageData output to the Data Repository and signals the Main Control.

The Parametric Image generation module follows the same structure and in essence is an extension of Spectral Analysis. The purpose of this module is to generate parametric maps based on each individual spectral parameter. The main difference between the two is that the Parametric Module uses a slightly modified algorithm to generate the parametric maps which is based on a sliding window algorithm. While the Spectral analysis computes averaged global values for each individual spectral parameter pertaining to the user selected ROI, the Parametric Module uses a smaller window of data points to compute the spectral values. The averaging performed on a large user selected ROI, can potentially misrepresent the true values of the spectral parameters due to a heterogeneous tissue composition. The sliding window algorithm allows the user to investigate and find the balance in the size of the averaged region. The larger the window size the more computationally expensive the image generation becomes. Furthermore the large window size increases the influence of data points residing 
further from the current calculation point. Figure 25 illustrates the main concepts used to generate parametric images from the spectral parameters. The first step in the generation of parametric images is the definition of the size for the sliding window by the user, referring to the number of scan lines ( $x$ direction) and number of data points per scan line (y direction) illustrated in Figure 25. The window is used to compute the spectral values pertaining to the position at the center of the window. Therefore, resulting parametric images generated are $2 x$ the widow size shorter in height and $2 x$ the window size smaller in width.The second step is the extraction of the data point required to compute the averaged power spectra for the window in the current position. The third step involves the normalization of the averaged power spectra with the reference power spectrum. The computation of the values for each individual spectral parameter, $\mathrm{MBF}, 0-\mathrm{MHz}$ intercept and spectral slope are extracted from the normalized power spectrum. The values are placed in separate data arrays corresponding to each individual parameter and the window changes position by one scan line in the $\mathrm{X}$ direction. Once the end of the scan lines has been reached, the sliding window changes position back to the first scan line and a window size length down in the $Y$ direction. Once the sliding window has traversed the entire image, the resulting parametric images are displayed for the user to apply custom color transfer functions. When the process is performed on an ultrasound volume, the sliding window repeats the process over to the next available frame.

The combination of Spectral Analysis Module with the Parametric Image Module creates a powerful platform allowing for a variety of applications in the Medical Biophysics field when spectral analysis is applicable. This platform has been used with success in performing pre and post treatment spectral analysis of tumors in mouse model xenographs. In the work presented in this thesis, this platform was used to perform spectral analysis and generate parametric maps of spectral parameters for low frequency ultrasound RF data obtained from patients with prostatic carcinomas. Another application where this platform is being utilized to perform spectral analysis and parametric map 
generation involves the treatment monitoring in a currently undergoing study of patients with locally advance breast cancer. The goal of the study is the classification, at the early stages of the treatment process, of patients responding to the treatment versus patients on which a particular treatment is not having an effect. 


\section{Figures}

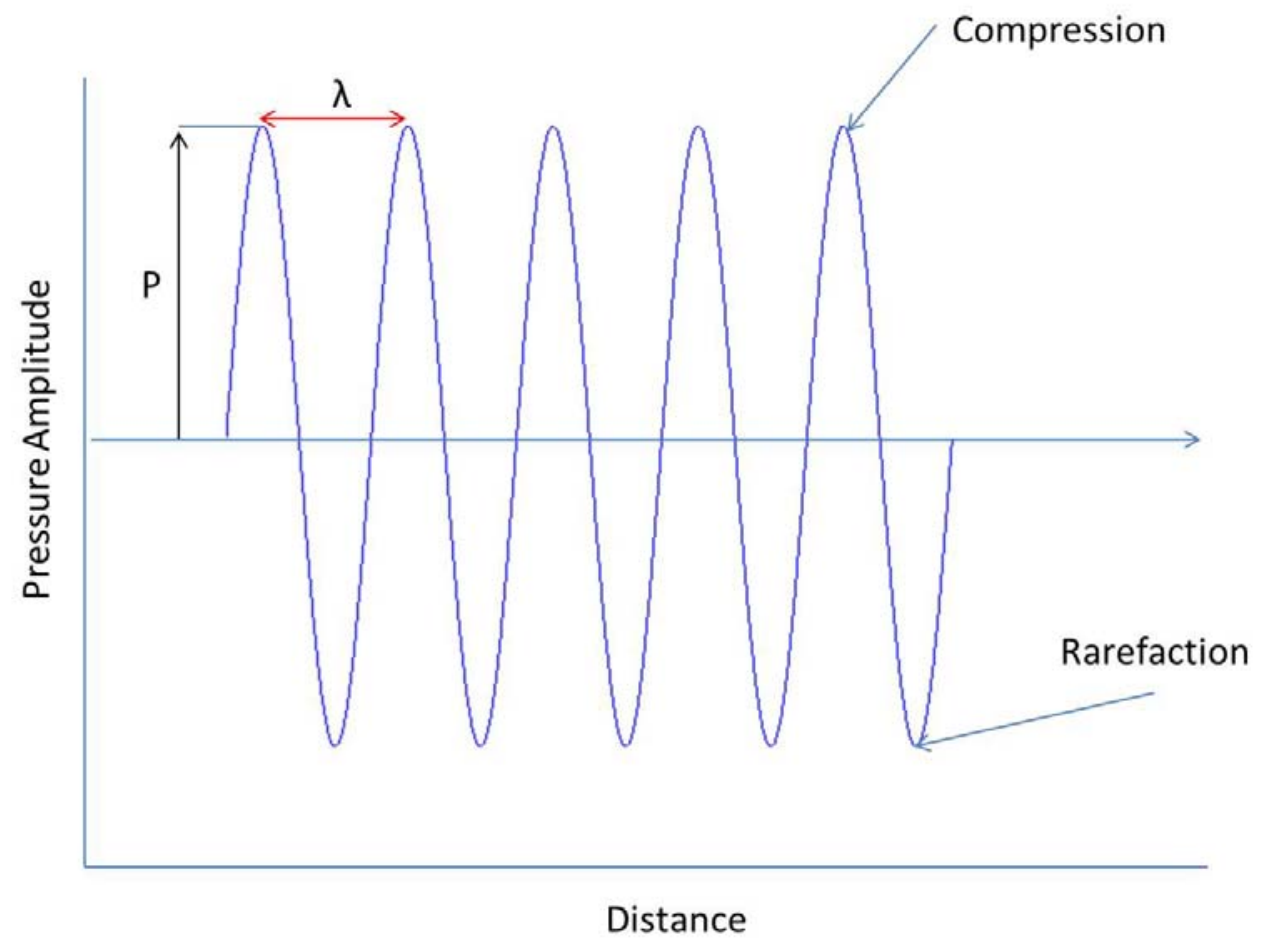

Figure 1: Sinusoidal waveform representation of a sound wave.

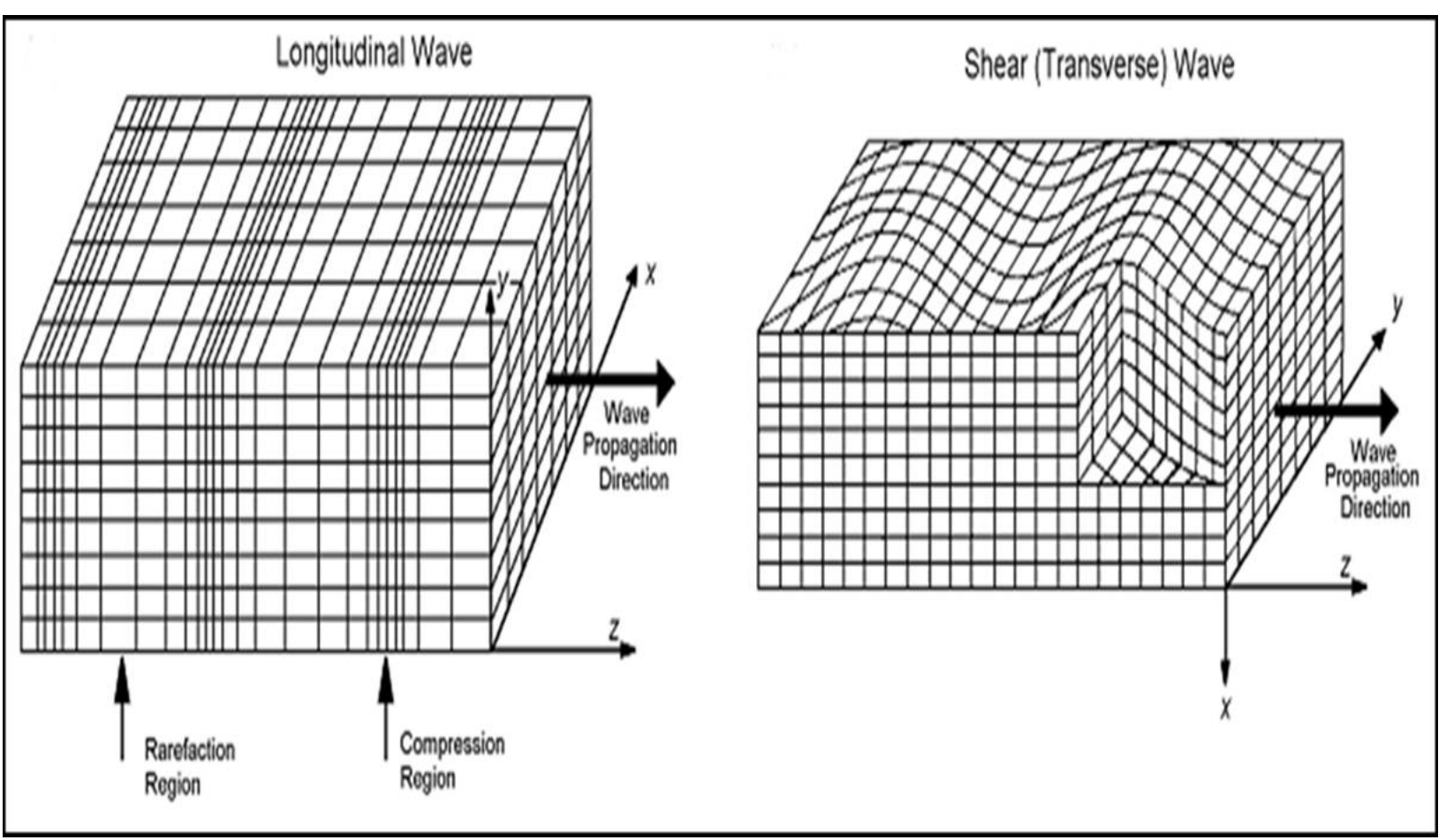


Figure 2: (Left) Longitudinal representation of a sound wave where a particle's motion (rarefaction and compression) is parallel with the direction of propagation. (Right) Shear wave representation of a sound wave where the particle motion is perpendicular to the direction of wave propagation.

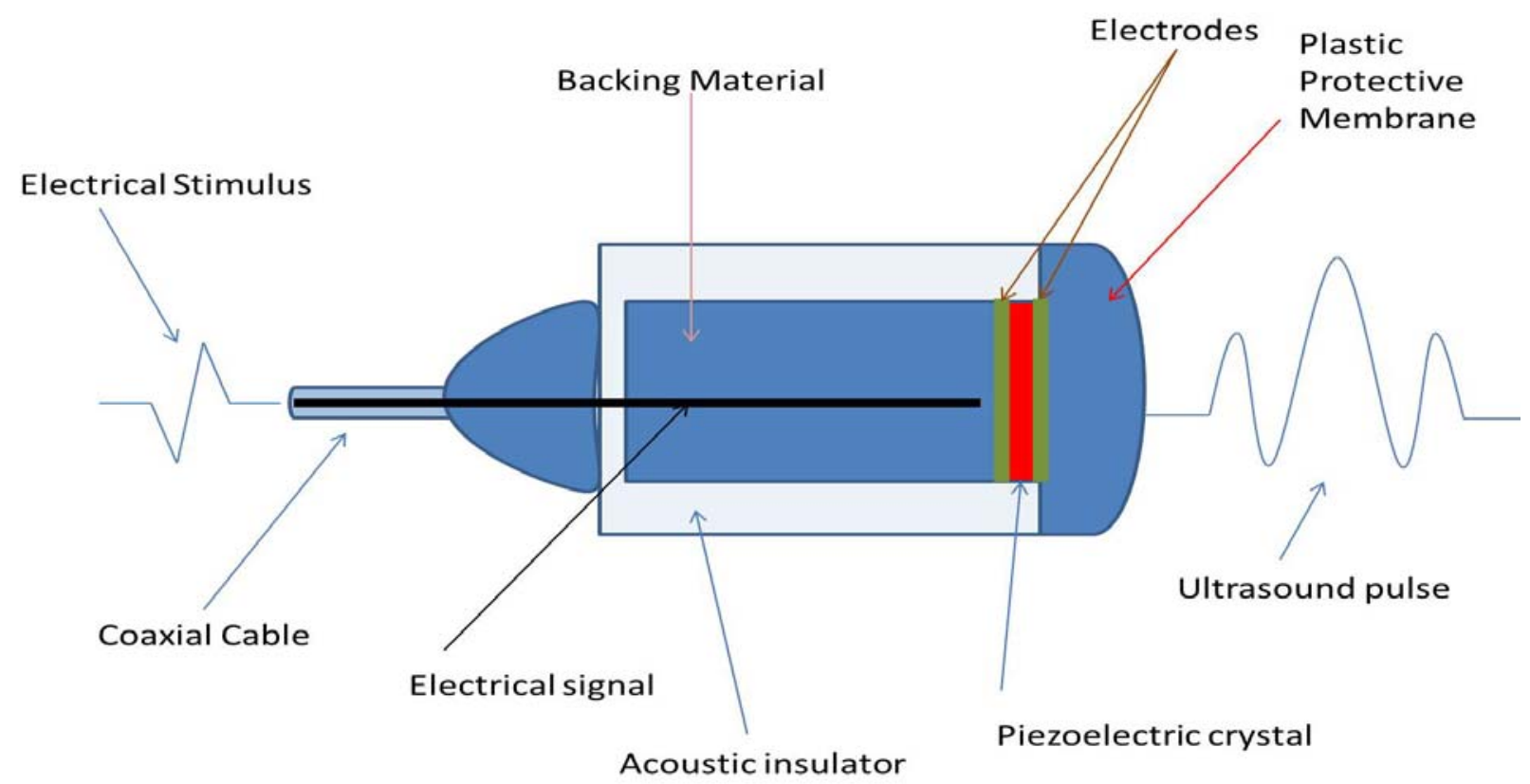

Figure 3: Pictorial representation of basic building components of an ultrasound transducer. Electrodes apply alternating potential difference causing a piezoelectric effect. As a result a piezoelectric crystal vibrates at high cyclical rates therefore converting the electrical energy into a mechanical sound wave. The result of the electrical input is a corresponding ultrasound pulse. 


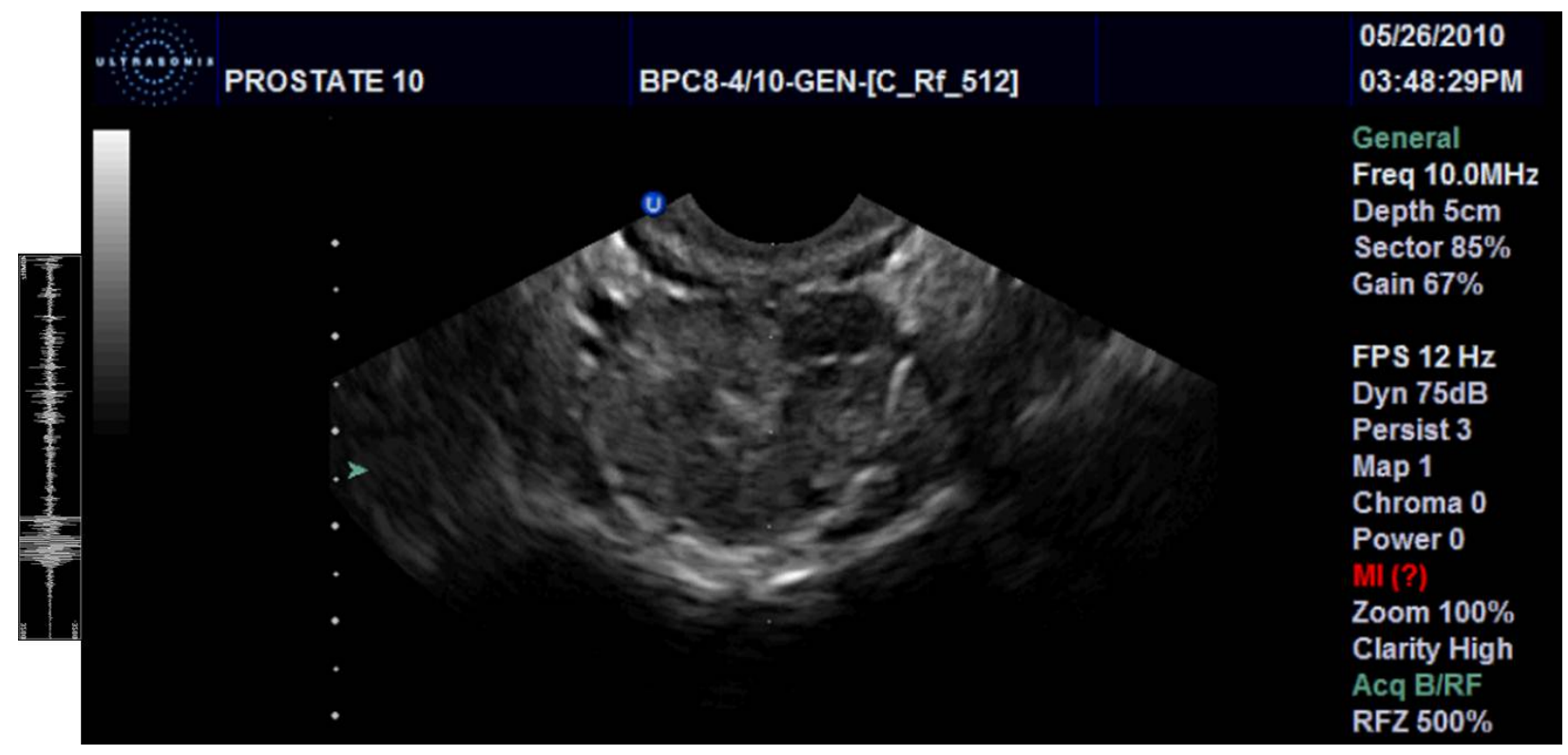

Figure 4: (Left) Representative A-mode trace prior to envelope detection through the middle of the prostate acquired using an Ultrasonix RP scanner with a transrectal probe. (Right) A typical transverse B-mode image of the prostate composed of multiple A-mode traces after signal envelope detection and processing to create a quality contrast two-dimensional image of the prostate gland. 


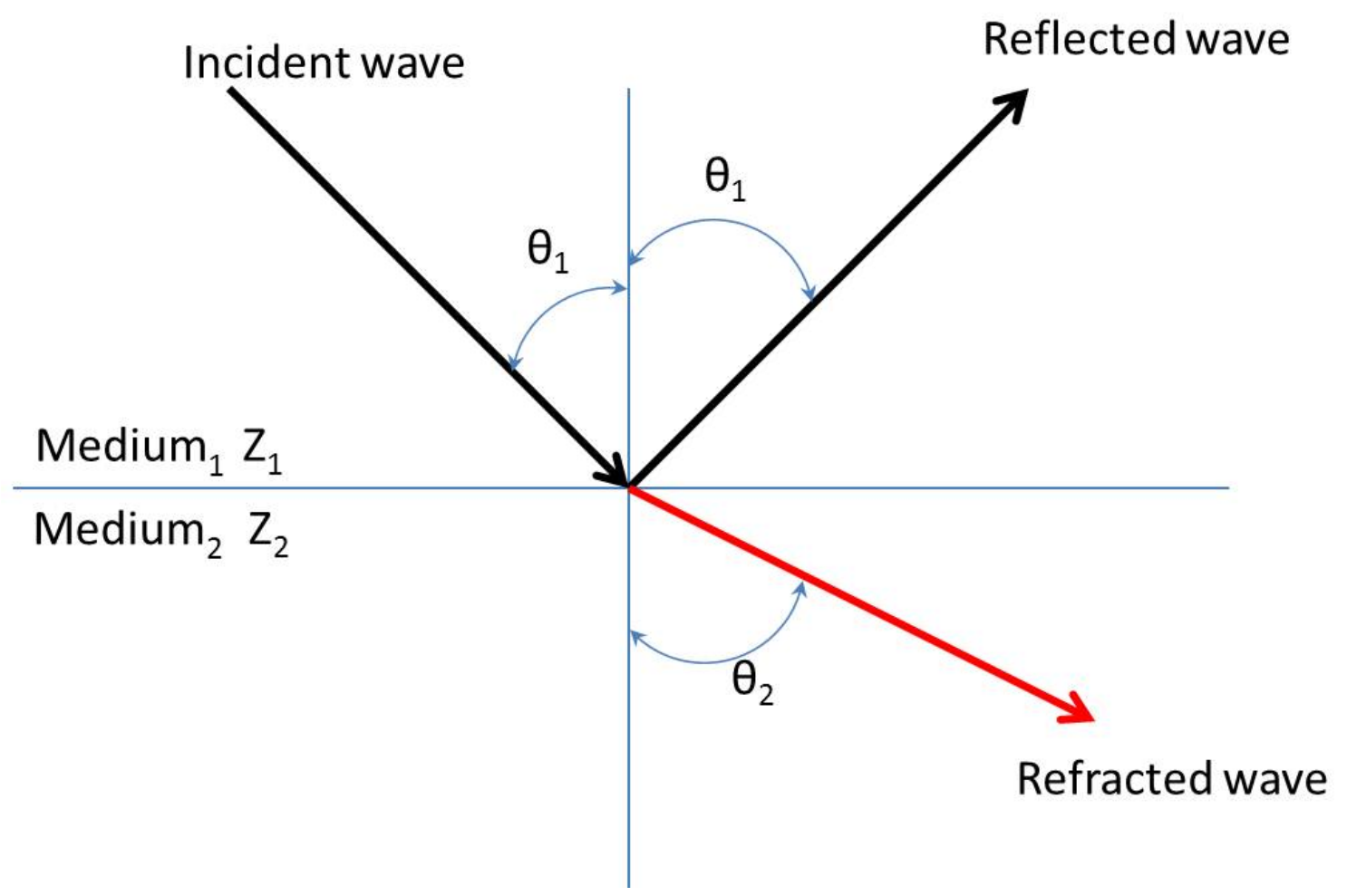

Figure 5: The interaction of the incident plane wave of wavelength smaller than the object which cause portion of the incident wave to reflect and refract. The ratio of the incident wave to the reflected wave requires knowing the acoustic impedance for both mediums $Z_{1}$ and $Z_{2}$ and is dependent on the incident angle $\theta_{1}$ and refraction angle $\theta_{2}$. 


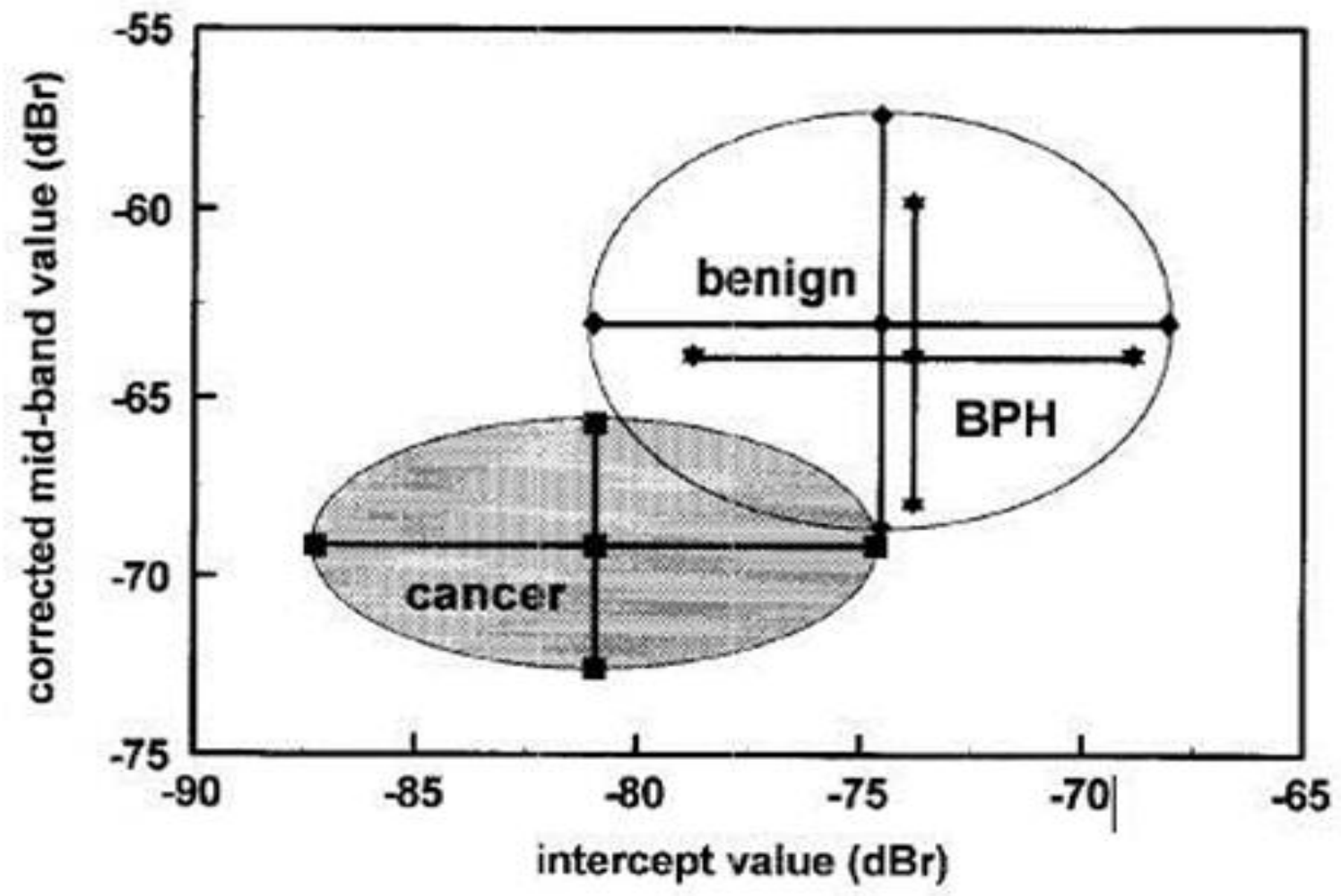

Figure 6: Values for the mean and standard deviation for spectral intercept and midband fit. These values represent the data from the learning set used in training the neural classifier. Cancerous set comprises of 29 independent ROI's . BPH and benign set comprises of a combined set of 60 independent ROI's. (Fellepa at al, Typing of Prostate Tissue by Ultrasonic Spectral Analysis, 1996)[30] 

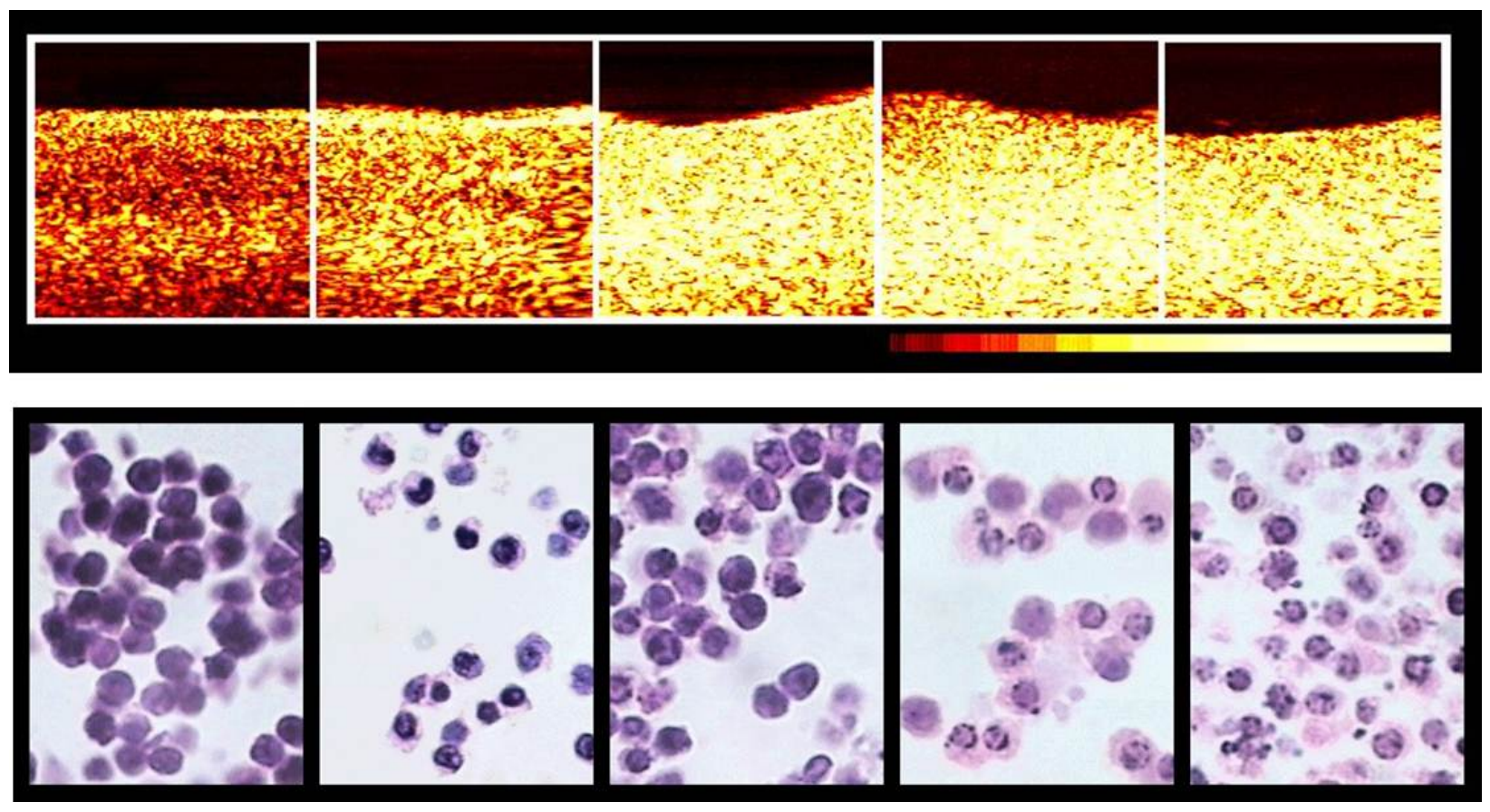

Figure 7: (Top) High-frequency ultrasound images of Acute Myeloid Leukemia (AML) cell pellets submerged into saline buffer then treated with chemotherapeutic agent cisplatinum for $0,6,12,24$ and 48 hours inducing a variable degree of apoptotic cell death. (Bottom) Representative corresponding histological analysis stained with H\&E, depicting the degree of apoptotic cells present in the pellet.(Czarnota et al, Ultrasound imaging of apoptosis: high-resolution non-invasive monitoring of programmed cell death in vitro, in situ and in vivo,1999) [35] 


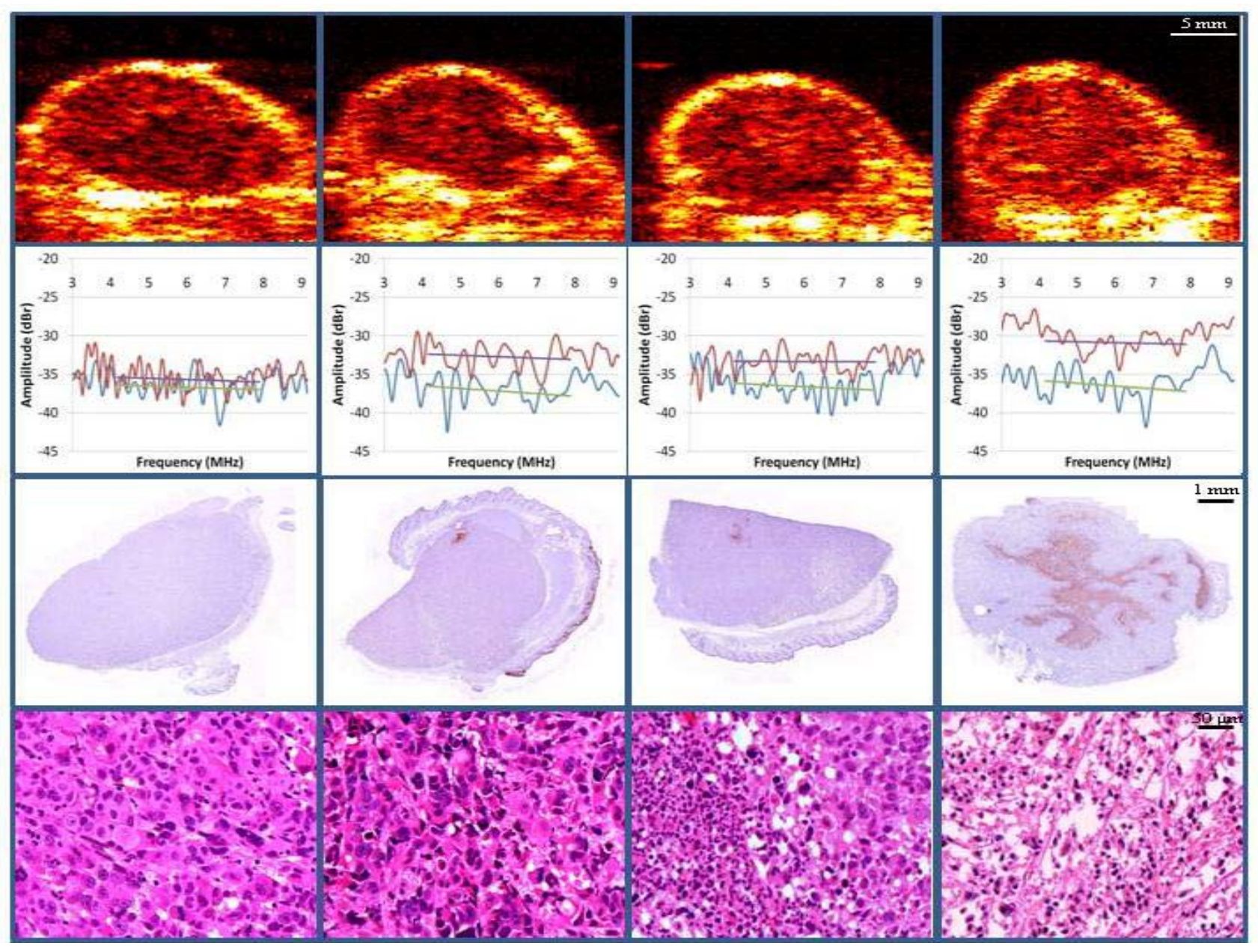

Figure 8: (First row) Representative low-frequency ultrasound images of PC3 tumors indicating variability in echogenicity depending on treatment regimen. In left to right order: untreated tumor, 8 Gy radiation, anti-vasculature microbubble treatment and $8 \mathrm{~Gy}$ radiation followed by , anti-vasculature microbubble treatment. (Second row) Normalized power spectra taken before treatment and 24 hours following treatment for each group. (Third row) Low magnification light microscopy images of TUNEL stained PC3 tumor slices. (Forth row) Light microscopy images of H\&E stained PC3 tumor slices at high magnification. (Papanicolau, Conventional frequency ultrasound detection of tumor response in vivo to cancer treatment administration,2009)[41] 


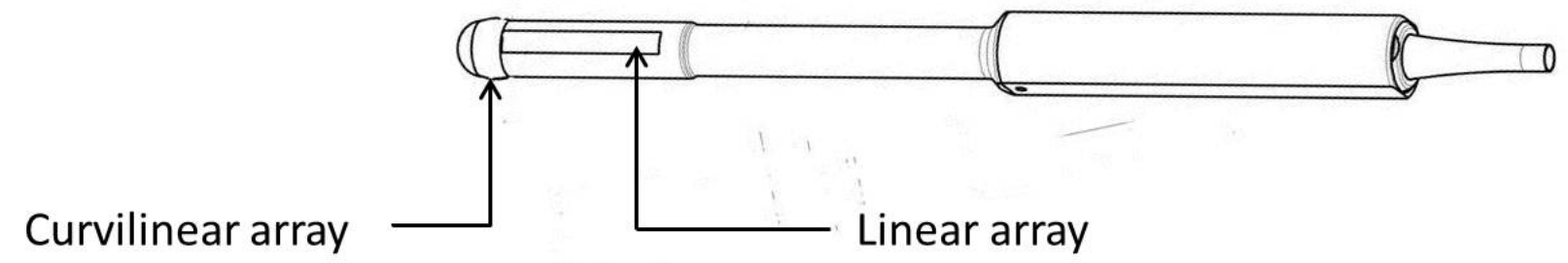

Figure 9: Schematic of the bi-planar transrectal probed used in this experiment. (Courtesy of Vermon S.A.) 


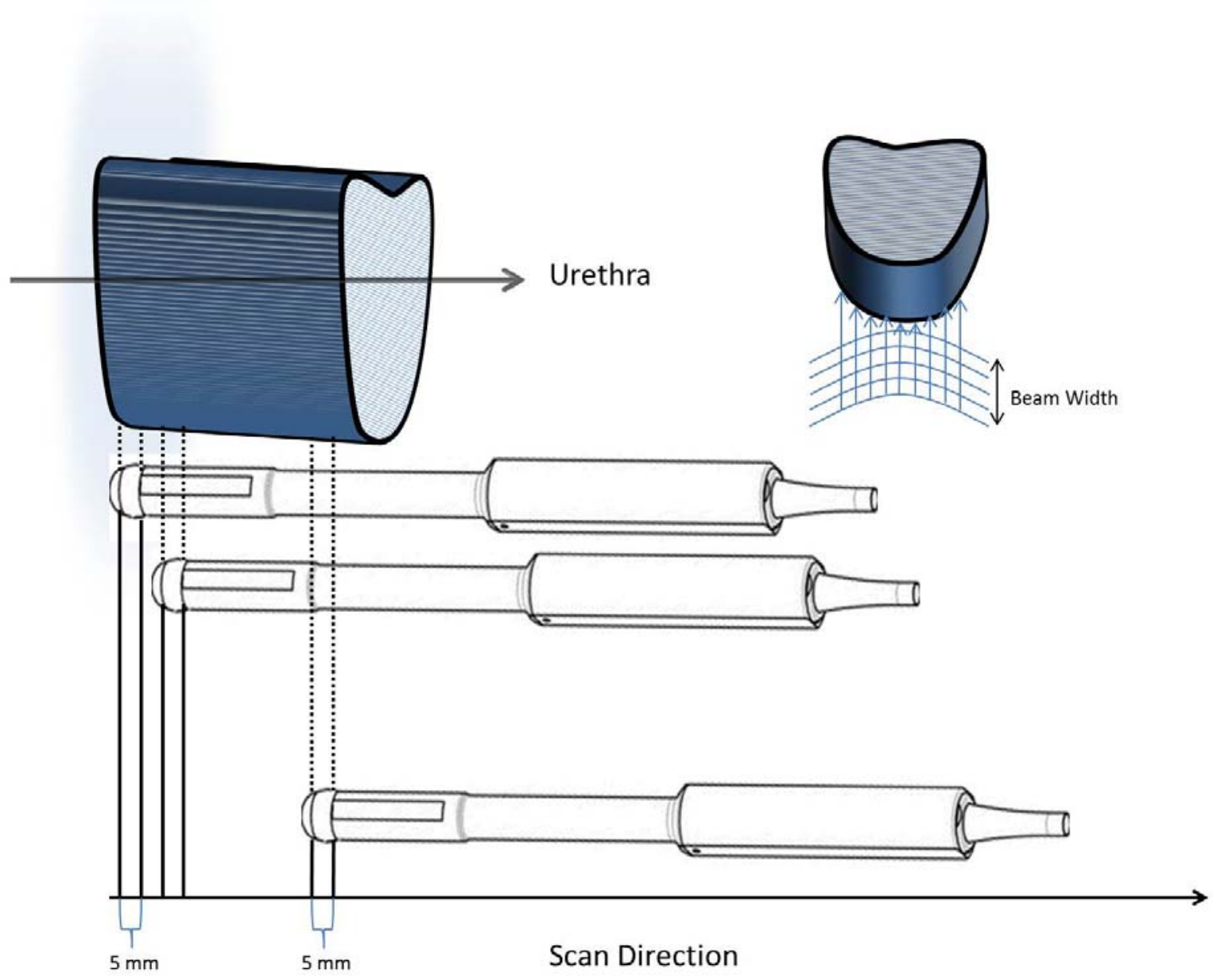

Figure 10: Schematic of the direction of the scan and the probes realive position to the prostate gland. 


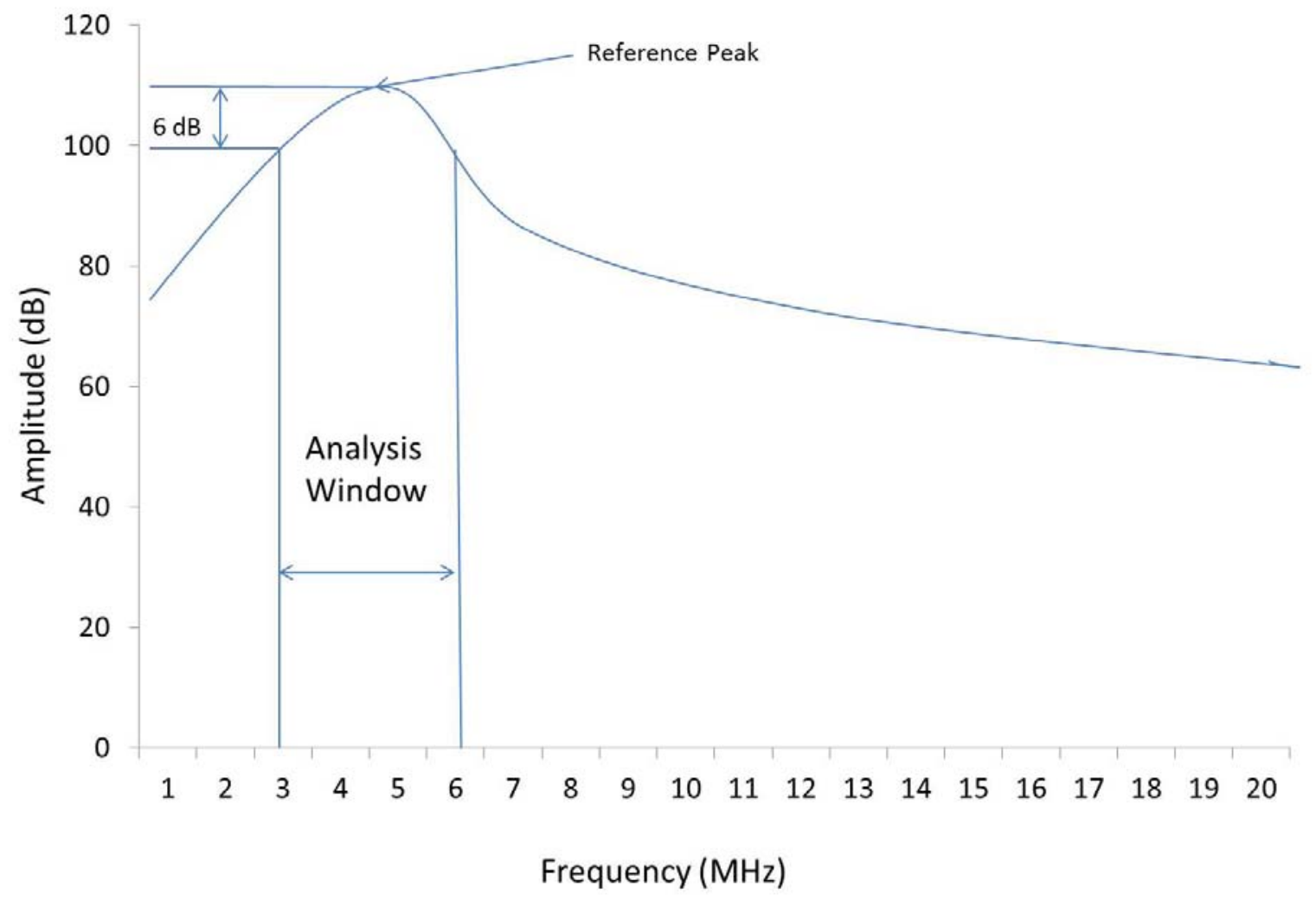

Figure 11: Depiction of $6 \mathrm{~dB}$ analysis window determined from the power spectra of the reference pulse. 


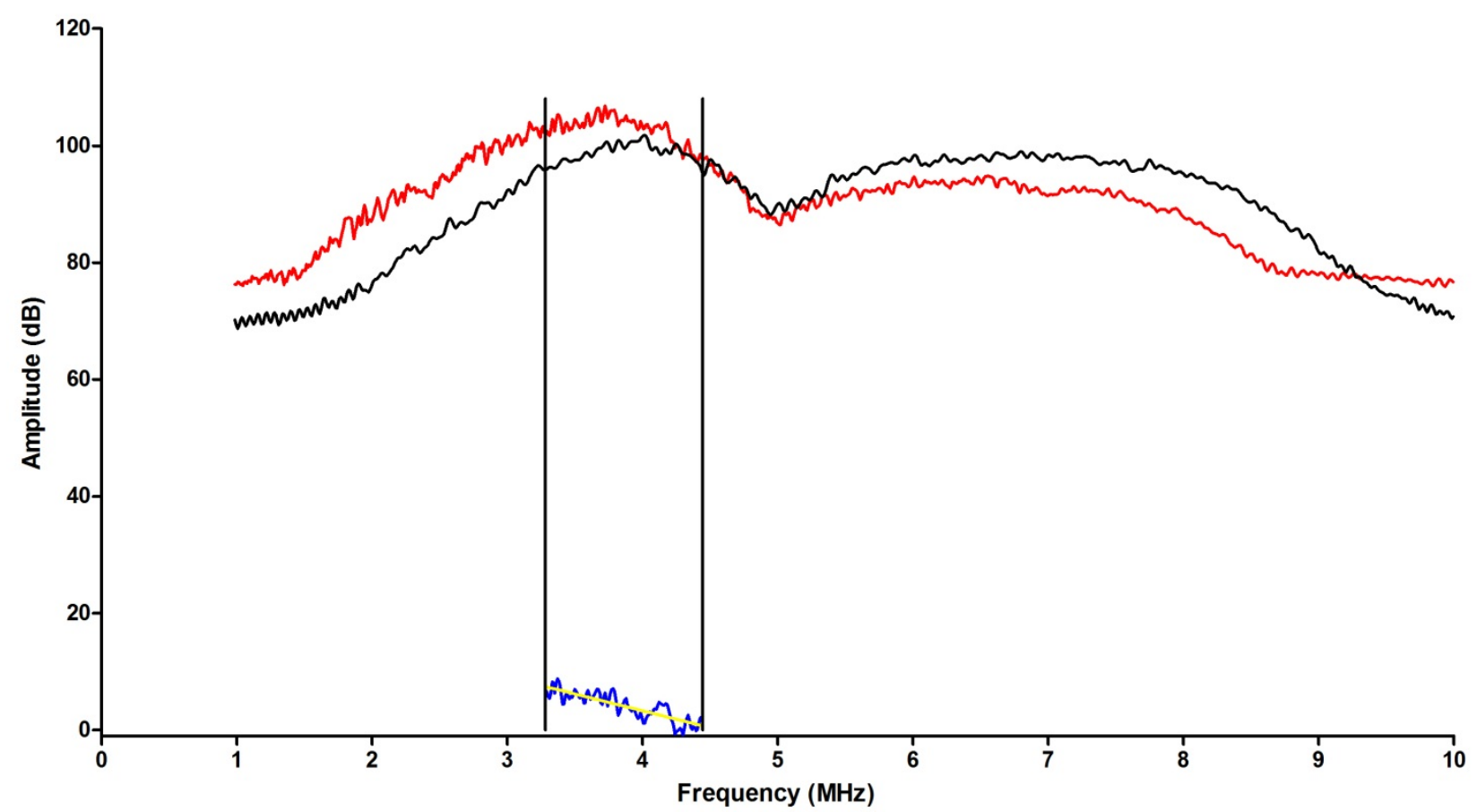

- ROI Power Spectrum

- Reference Power Spectrum

- Normalized Power Spectrum - Line of Best Fit

Figure 12: The red curve is a representative power spectra corresponding to an ROI representative of the entire prostate gland. The black curve is a power spectra collected from a phantom to be used for normalization, taken with the same machine settings as the one collected from tissue. The blue curve is the resulting normalized power spectra obtained from the subtraction of the tissue power spectra by the phantom power spectra. The linear regression is shown with the yellow line corresponding to the $6 \mathrm{~dB}$ analysis window. 


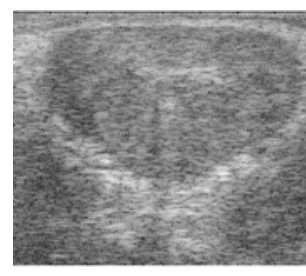

B-MODE

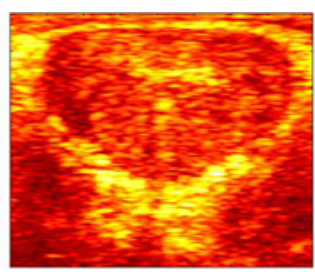

MBF

Parametric Image

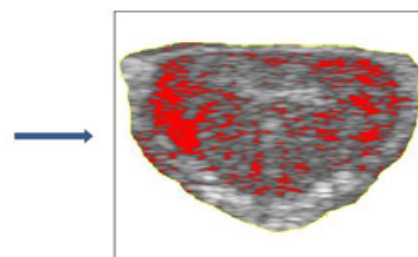

Manual Segmentation \& Thresholding

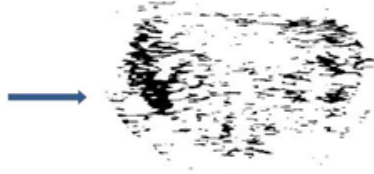

Extracted

Areas of Suspicion

Figure 13: The main steps followed in our analysis mechanism are illustrated here starting with the B-mode reconstruction from raw RF data shown on the left most of the image. The second step is the generation of the parametric images for each of the three spectral parameters under investigation. The second image is a representative parametric image generated using the mid-band fit parameter. The second last image is the corresponding image of the manual segmentation of the parametric image in which only the areas of the prostatic gland are included. Also in this stage the threshold value is discovered and in turn applied to all the parametric slides for each prostate. In the last phase only the thresholded areas where the MBF parameter suggests high likelihood of carcinoma presence in the prostate are extracted. The areas within the bounds of the prostate still present in the image are then added to compute the total are of suspicion. 

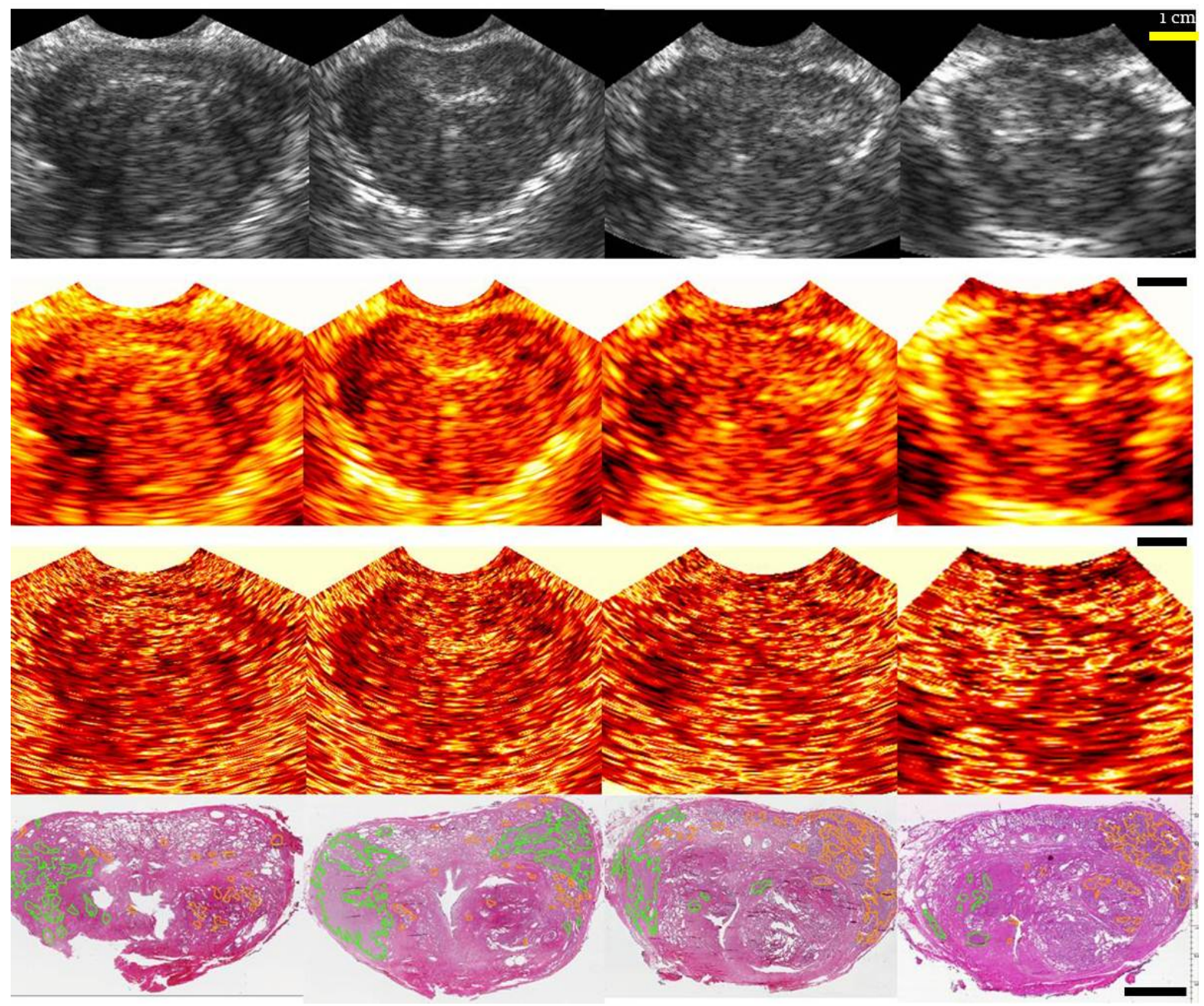

Figure 14: Top row displays ultrasound images reconstructed from RF data. Each individual image represents a stationary plane through the prostate in the direction base to apex. All the images shown are obtained from one representative patient representing 4 acquisition steps which are separated by a $5 \mathrm{~mm}$ gap in between. The second row displays the resulting parametric images of the MBF parameter from the same planes obtained as a result of spectral analysis. The third row displays the resulting parametric images of the spectral intercept obtained from the same planes. The last row displays the corresponding slices obtained from histological analysis. The histology slices are representative $\mathbf{4}$ um slices obtained from $\mathbf{5}$ $\mathrm{mm}$ blocks which correspond to the same regions for which the ultrasound RF data was collected. On the histology images areas of prostatic carcinoma are contoured with green color. Areas of abnormal glandular activity are contoured in orange. 

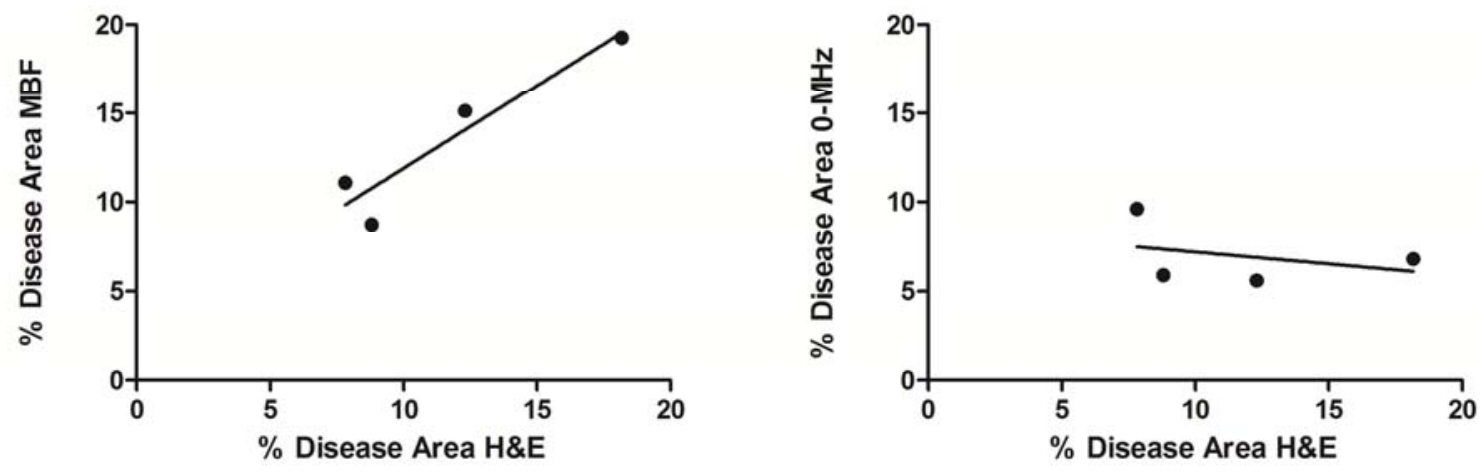

Figure 15: (Left) A graphical representation of the MBF percent area versus the corresponding percent area from the histopathological findings for all the steps collected from Patient 1. A linear regression analysis is showing with the solid line. (Right) The 0-MHz spectral intercept versus the hisptopathological analysis corresponding to the percent disease area as well as a linear regression noted with a solid line. 


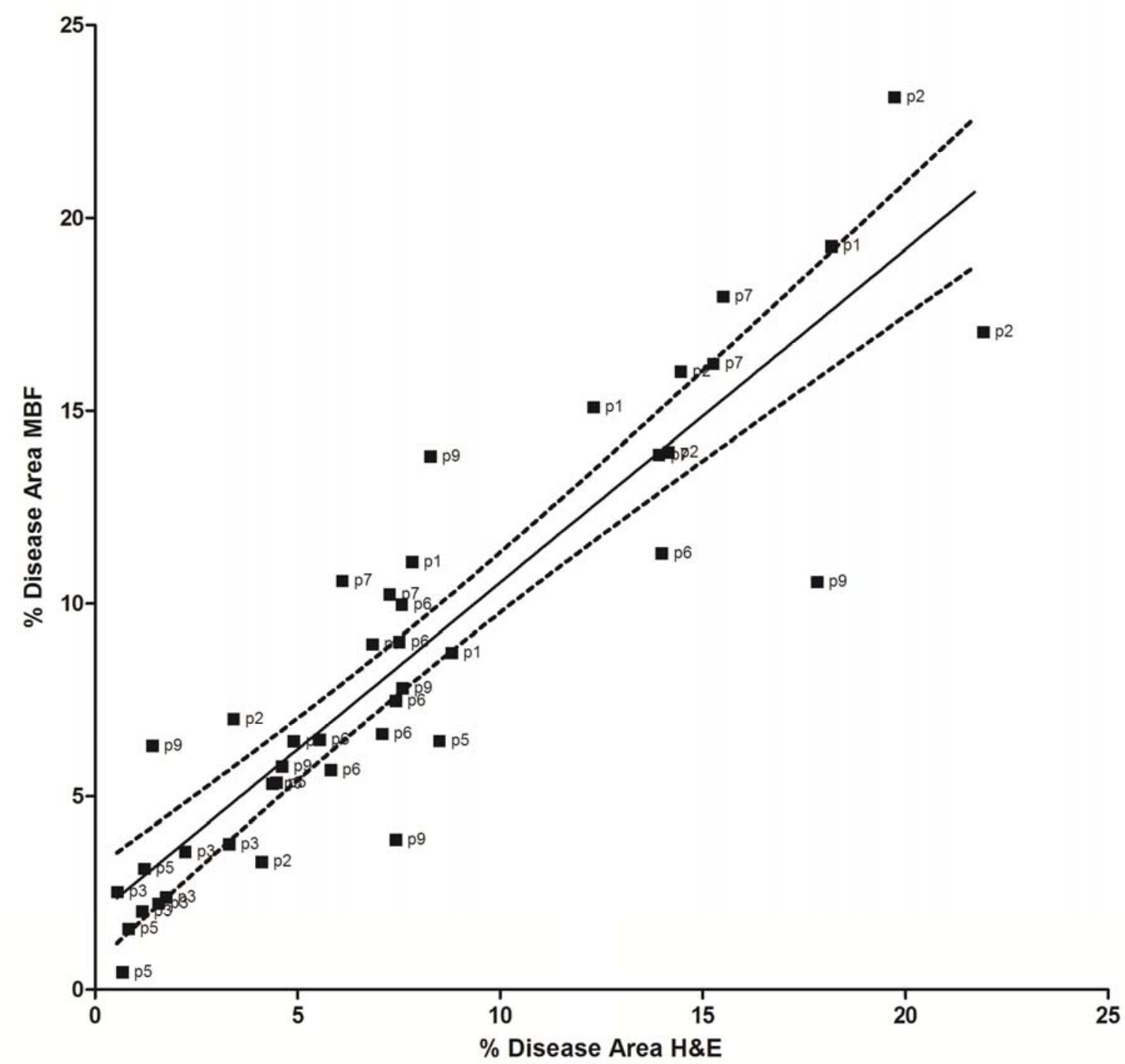

Figure 16: Cumulative scatter plot depicting the percent disease area obtained from MBF parameter versus the percent disease area obtained from histological analysis. The data shown in this graph represents all the data pairs from individual steps collected for the entire patient population which participated in the study. The solid line represents the best fit line obtained from liner regression with the corresponding $95 \%$ confidence interval is depicted with a broken line. 


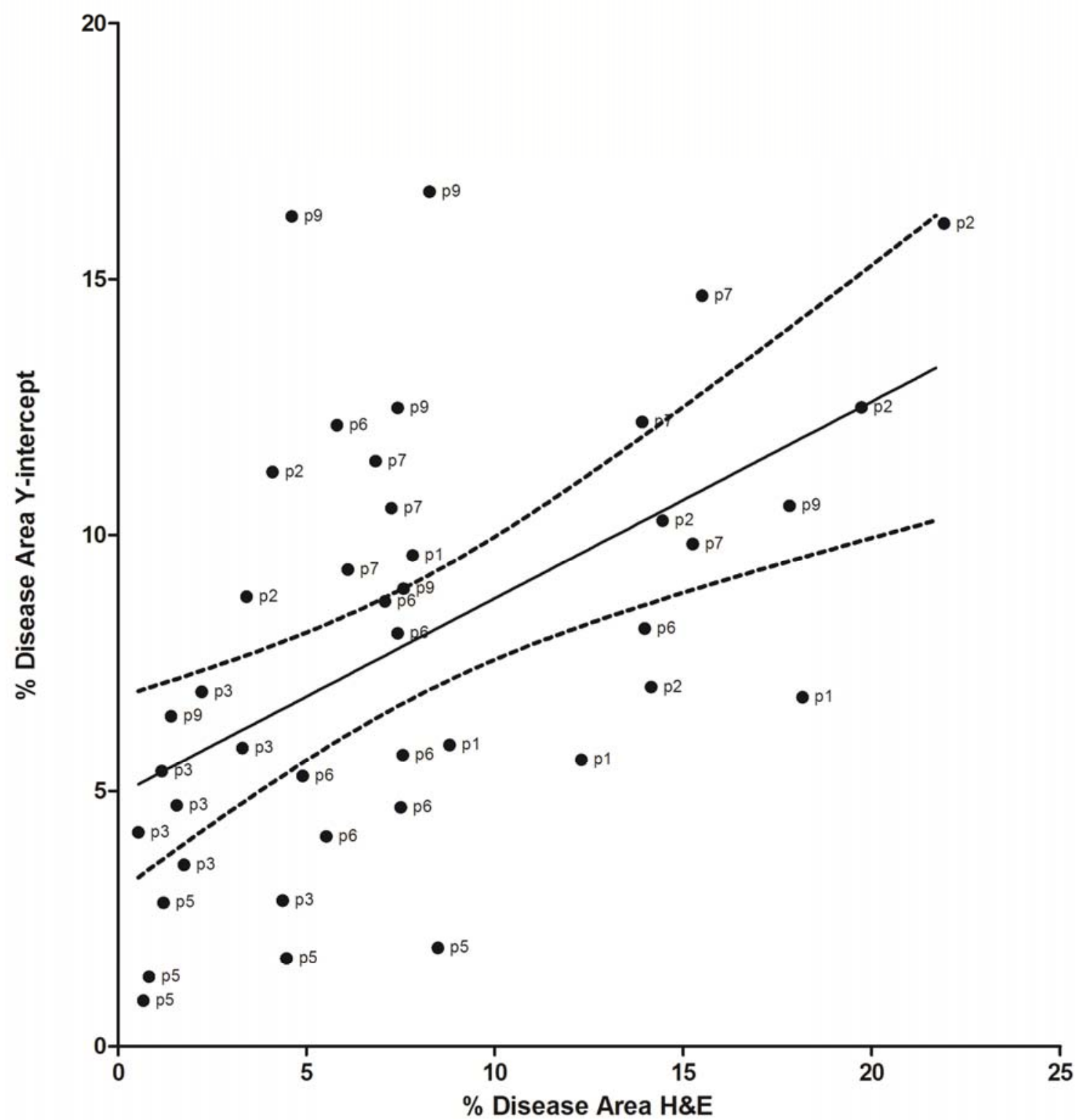

Figure 17: Cumulative scatter plot depicting the percent disease area obtained from the spectral intercept parameter versus the percent disease area obtained from histological analysis. The data shown in this graph represents all the data pairs from individual steps collected for the entire patient population which participated in the study. The solid line represents the best fit line obtained from linear regression with the corresponding $95 \%$ confidence interval depicted with a broken line. 


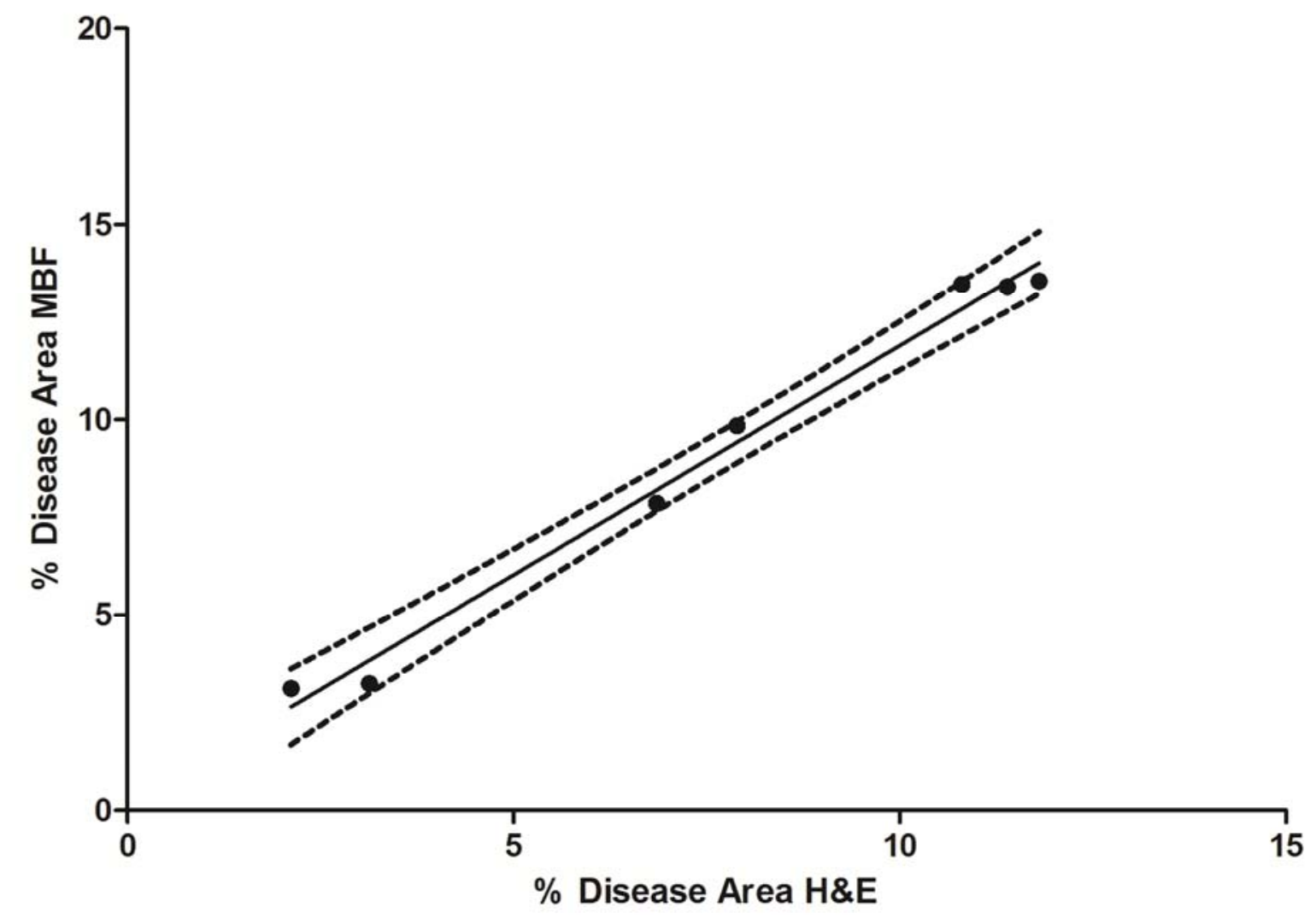

Figure 18: In this graph the each point represents a patient who participated in our study. The Y-axis shows the average percent disease area computed from all the slides belonging to each individual patient. The X-axis is the average percent disease area calculated by adding the results of histopathological analysis of each slide pertaining to each individual patient. The solid line represents the linear regression goodness of fit with the $95 \%$ confidence interval shown in the broken line. 


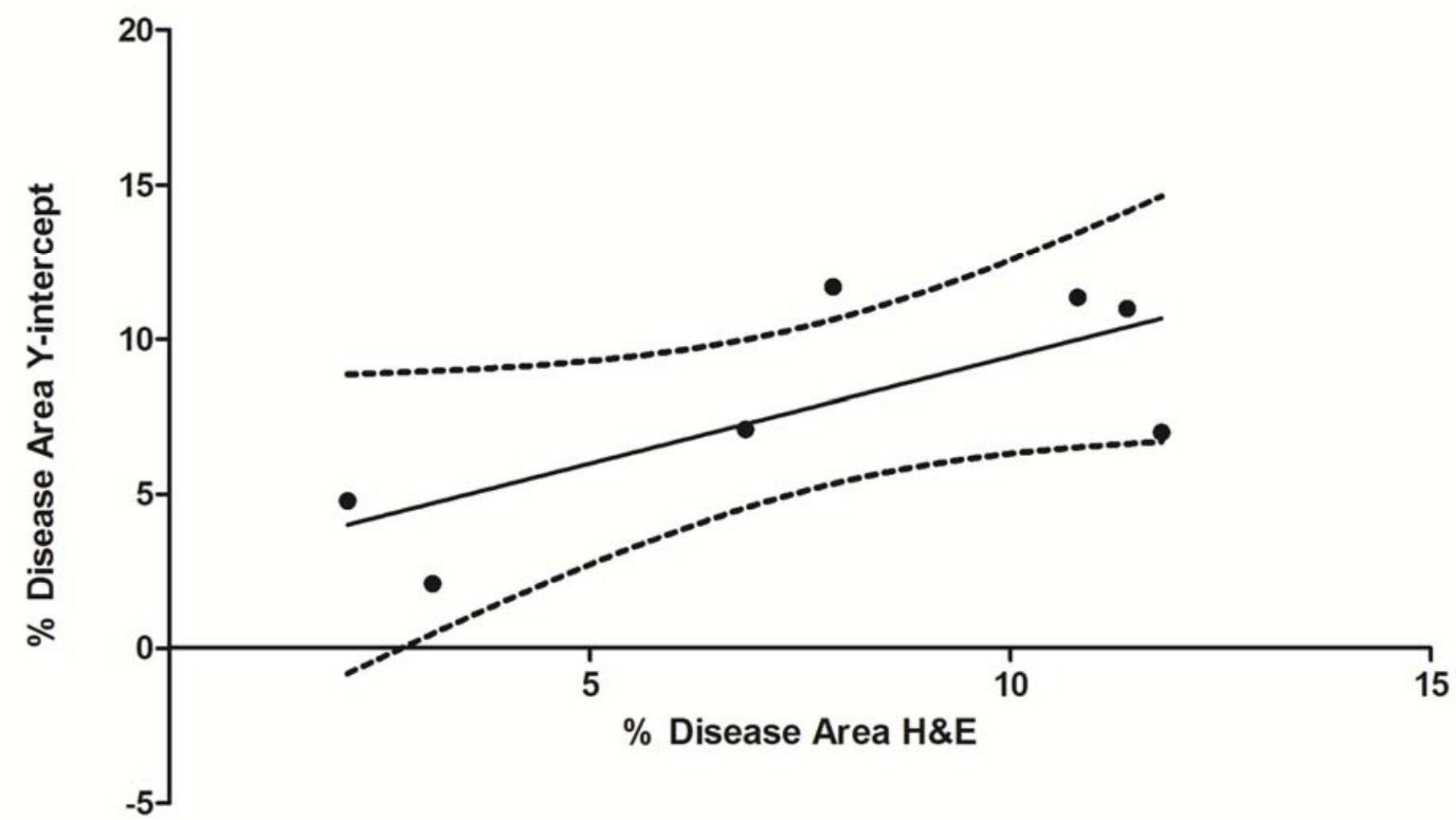

Figure 19: In this graph the each point represents a patient who participated in or study. The Y-axis shows the average 0-MHz intercept percent disease area computed from all the slides belonging to the patient. The X-axis is the average percent disease area calculated by adding the results of histopathological analysis of each slide pertaining to the patient. The solid line represents the linear regression goodness of fit with the $95 \%$ confidence interval shown in the broken line. 


\section{Application}

\section{Application (Win 32 part)}

\section{Application (.NET part)}

\section{vtkDotNetWrapper}

\section{VTK (Win 32)}

\section{.NET Framework}

\section{Windows}

\section{Hardware}

Figure 20: High level conceptual model of application architecture depicting the various layers and their scope. The vtkDotNetWrapper acts a middleware layer of communication joining the native .NET framework with the VTK win32 librariers. 


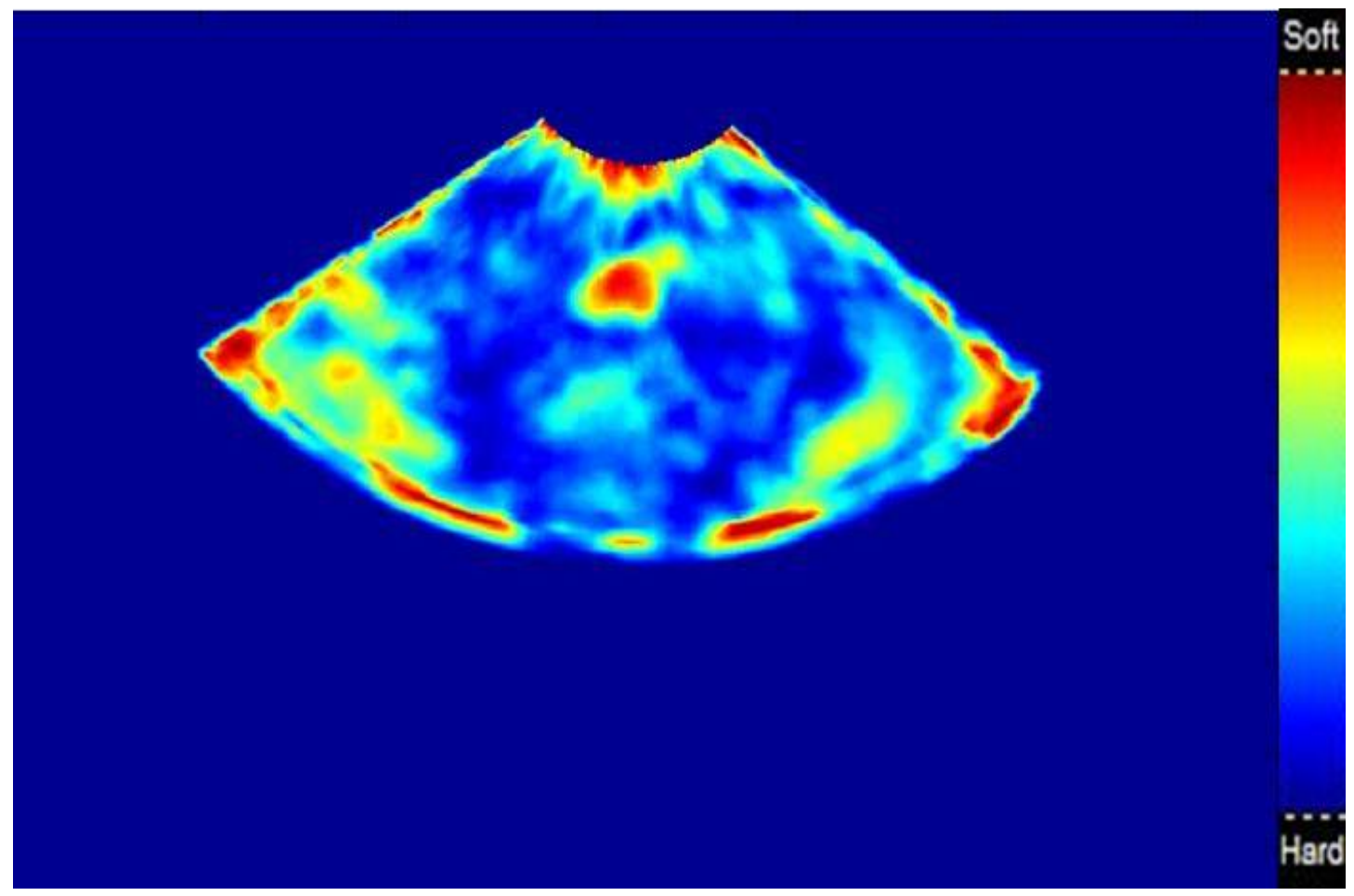

Figure 21: Representative elastrogram obtained from the same patient shown in Figure 14. The elastogram was obtained using Ultrasonix RP ultrasound system with a curvilinear transrectal transducer pulsed at $10 \mathrm{MHz}$ with a sampling frequency of $20 \mathrm{MHz}$. The color bar on the right hand side shows the color distribution corresponding to the relative tissue stiffness distribution. 


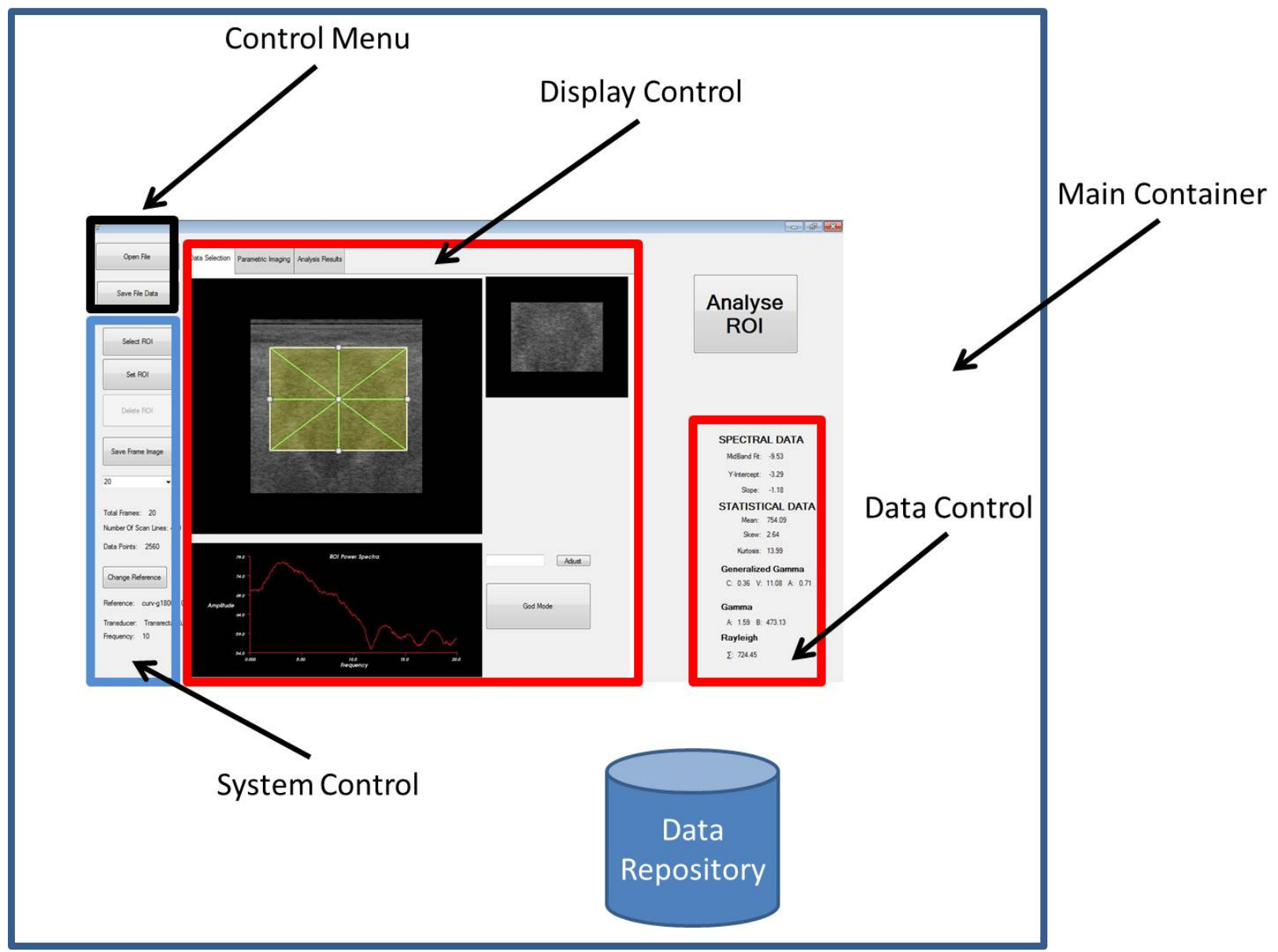

Figure 22: A visual representation of the various components comprising of the Spectral Analysis Module. Each individual component communicates with the rest through a system of delegates passing through the Main Container. 


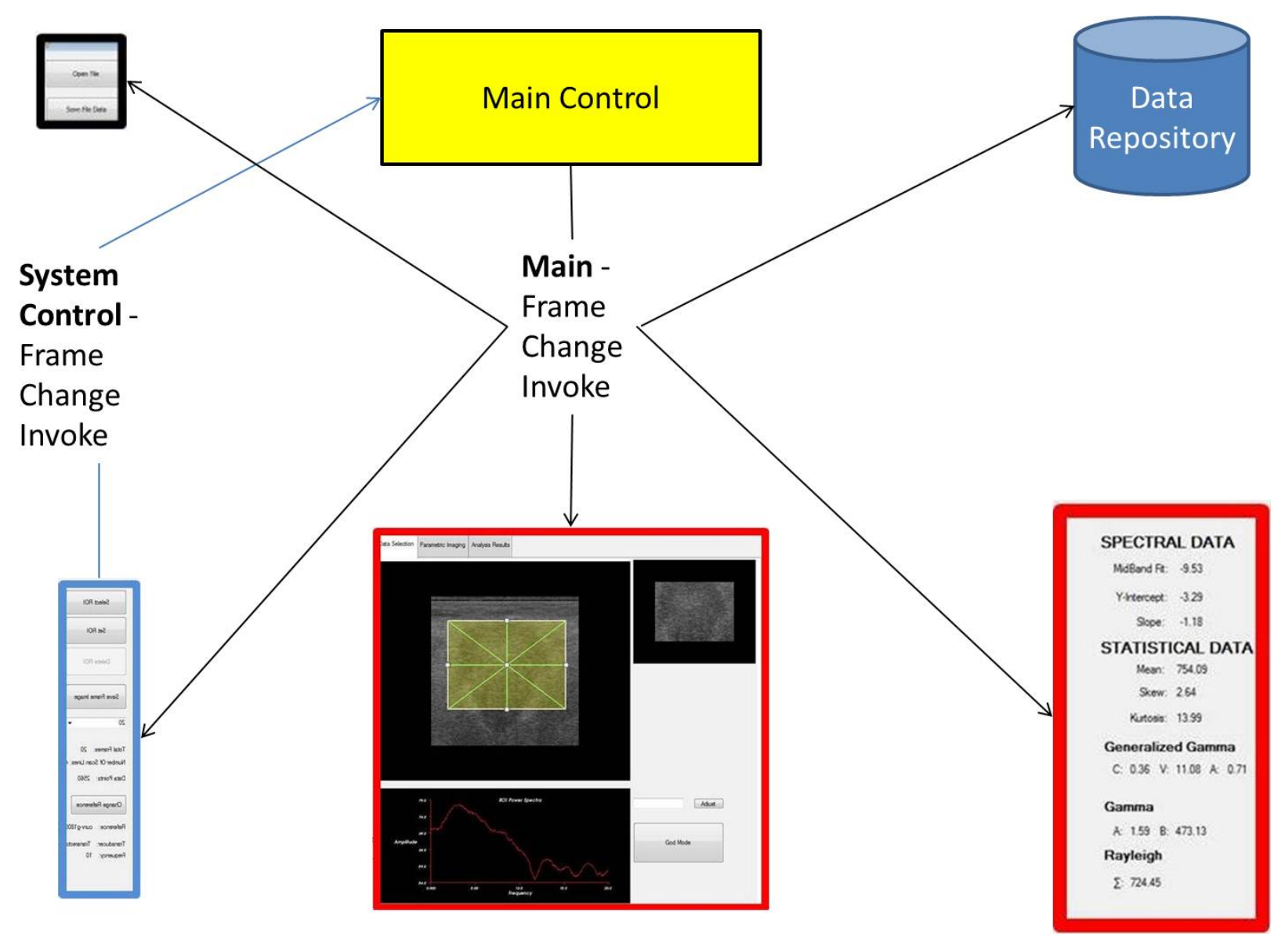

Figure 23: An illustration of the communication pathways between the various components in the Spectral Analysis Module. In this scenario, the System Control invokes a frame change event ( the frame number from an multi-frame ultrasound file) with the Main Control. Since, all the available components are registered with the Main Control, the Main invokes a global change frame event. 


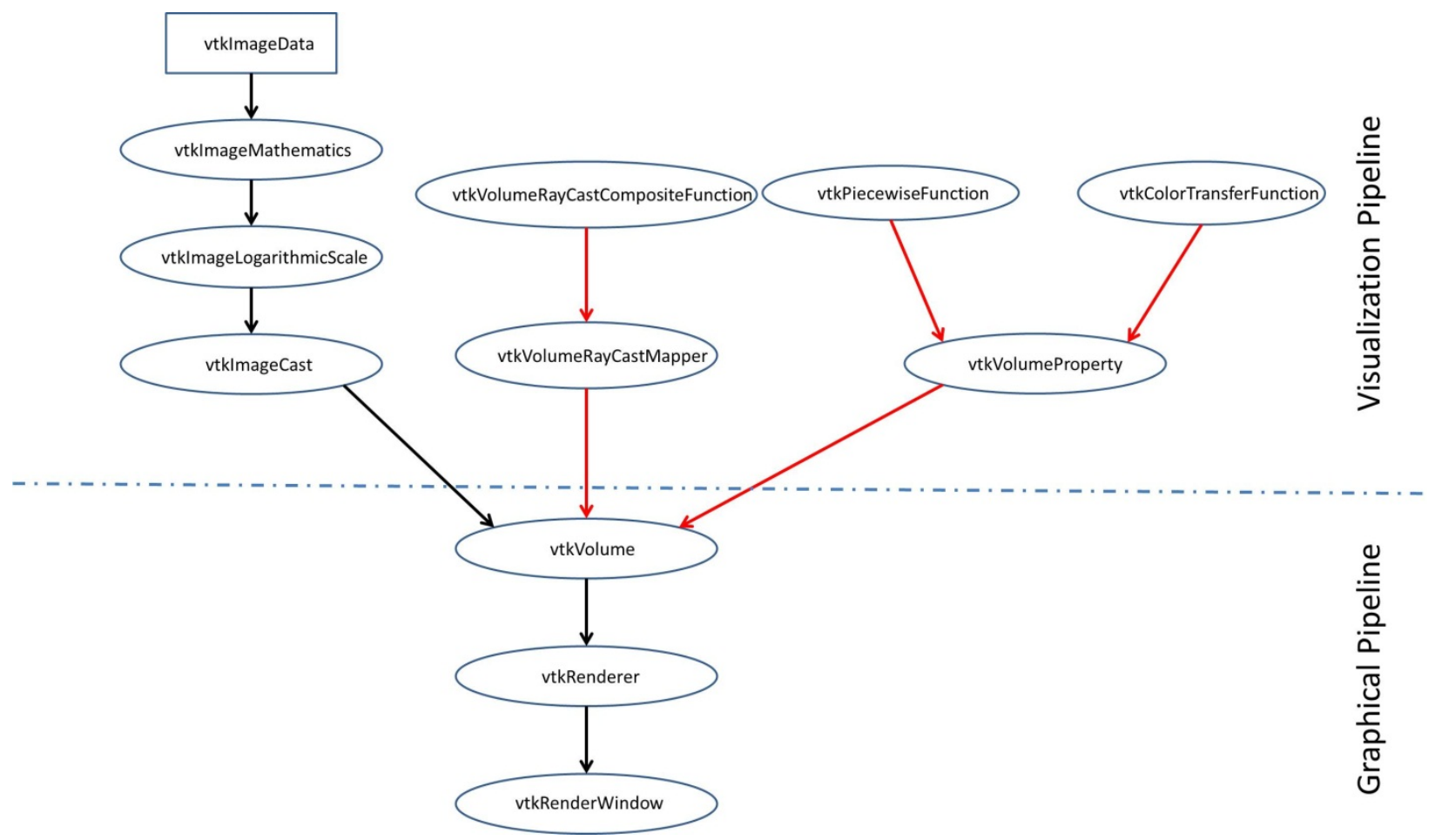

Figure 24: Visual representation of the display pipeline utilized by the Display Control component. The visualization pipeline above the dashed line shows the VTK objects utilized in the visualization process prior to rendering. The red arrows delineate the classes which define how the dataset will be visualized. These objects can encompass various filters and functions which define the visualization properties of an object and may include color, opacity, lighting, texture, etc. The path shown with black arrows shows the path which the dataset takes from the basic structure through the various filters which change the state of the data to create the volume prior to rendering. Bellow the dashed line is the graphical pipeline. Once the volume object is created and the visualization properties are added, the volume object undergoes through the rendering process. Finally the result of the rendering process is displayed by the rendering window. 

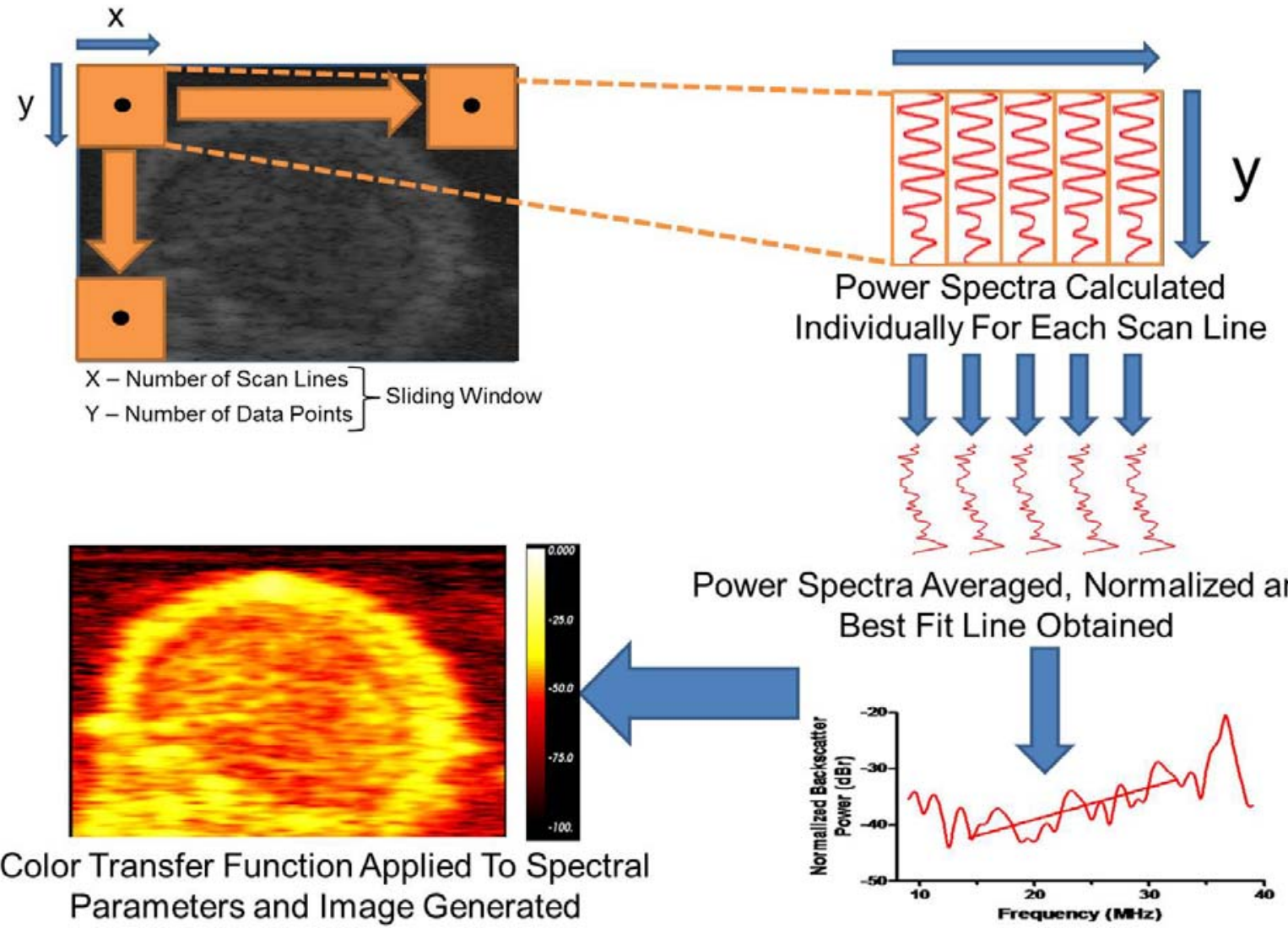

Figure 25: An illustration of the steps taken in generating parametric images from spectral parameters using a sliding window algorithm. Step 1 defines the size of the sliding window, referring to the number of scan lines ( $x$ direction) and number of data points per scan line ( $y$ direction). The window is used to compute the spectral values pertaining to the position at the center of the window. The second step is the extraction of the data point required to compute the averaged power spectra for the window in the current position. The third step involves the normalization of the averaged power spectra and computation of the values for each individual spectral parameter, MBF, 0-MHz intercept and spectral slope. The values are placed in separate data arrays and the window changes position by one scan line in the $\mathrm{X}$ direction. Once the end of the scan lanes has been reached, the sliding window changes position back to the first scan line and window size down in the $\mathrm{Y}$ direction. Once the sliding window has traversed the entire image, the resulting parametric images are displayed for the user to apply custom color transfer functions. When the process is performed on an ultrasound volume, the sliding window repeats the process over to the next available frame. 


\section{Tables}

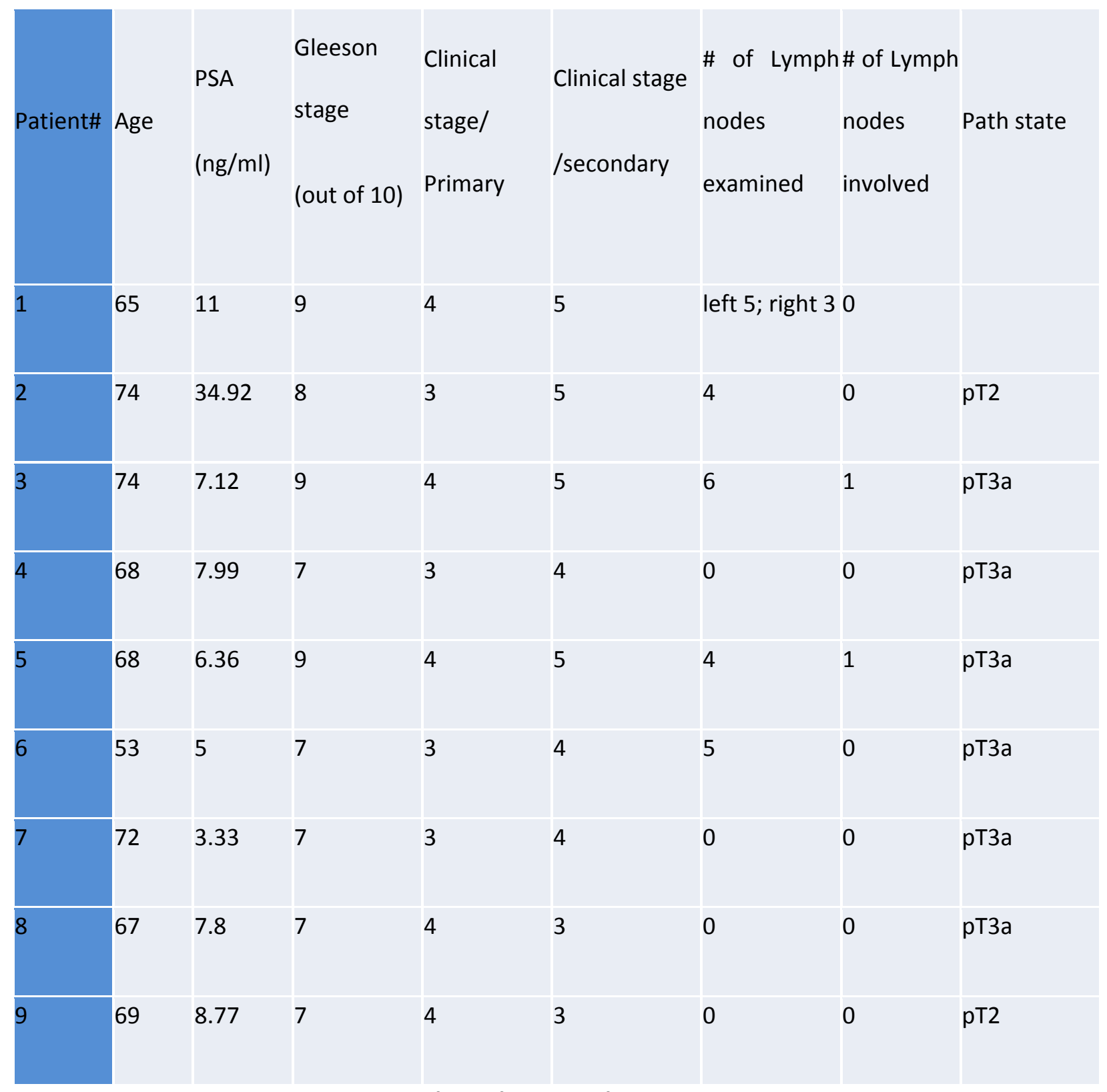

Table 1 - Clinical relevant patient characteristics for the first group of participants in the study. 


\begin{tabular}{|l|l|l|}
\hline & Curvilinear Array & Linear Array \\
\hline Center Frequency & $6.5+/-10 \% \mathrm{MHz}$ & $7+/-10 \% \mathrm{MHz}$ \\
\hline Number of Elements & 128 & 128 \\
\hline Pitch & & $0.42 \mathrm{~mm}$ \\
\hline Curvature Radius & $0.205 \mathrm{~mm}$ & none \\
\hline Elevation & $10 \mathrm{~mm}$ & $5 \mathrm{~mm}$ \\
\hline Bandwidth & $7 \mathrm{~mm}$ (measured $4.5 \mathrm{~mm}$ ) & none \\
\hline & & $20 \mathrm{~mm}$ \\
\hline
\end{tabular}

Table 2 - Acoustic specifications for the bi-planar transrectal probe, comprising of two linear array transducers. (Courtesy of Vermon S.A. and Ultrasonix) 


\begin{tabular}{|l|l|l|l|}
\hline \multicolumn{2}{|l|}{ Percent Disease Area (Patient 1) } & MBF & -MHz \\
\hline Slice \# & H\&E & 11.08 & 9.67 \\
\hline 1 & 7.82 & & 6.83 \\
\hline 2 & 18.17 & 19.3 & 5.6 \\
\hline 3 & 12.3 & 15.1 & 5.9 \\
\hline 4 & 8.8 & 8.73 & 7.0 \\
\hline Average & 11.8 & 13.55 & \\
\hline
\end{tabular}

Table 3 - Summarized results from Patient 1. The slice number represents the probes position as it moves away from the base of the prostate. The H\&E column is the result of the histological analysis preformed by a pathologist, which is used as our gold standard for our results are compared against it. 


\section{Bibliography}

[1] S. American Cancer, "American Cancer Society.: Cancer Facts and Figures 2010," ed, 2010.

[2] B. S. W. Catalona Wj, "Nerve-sparing radical prostatectomy: evaluation of results after 250 patients," The Journal of Urology, vol. 3, pp. 538-543, 1990.

[3] H. Zincke, et al., "Radical prostatectomy for clinically localized prostate cancer: long-term results of 1,143 patients from a single institution," Journal of clinical oncology : official journal of the American Society of Clinical Oncology, vol. 12, pp. 2243-2245, Nov 1994.

[4] W. Duncan, et al., "Carcinoma of the prostate: results of radical radiotherapy (1970-1985)," International journal of radiation oncology, biology, physics, vol. 2, pp. 365-367, 1993.

[5] M. B. Garnick, "Prostate cancer: screening, diagnosis, and management," Annals of internal medicine, vol. 10, pp. 804-818, 1993.

[6] G. Sandblom, et al., "Clinical consequences of screening for prostate cancer: 15 Years follow-up of a randomised controlled trial in Sweden," European Urology, vol. 46, pp. 717-723, 2004.

[7] D. S. Smith, et al., "Use of lower prostate specific antigen cutoffs for prostate cancer screening in black and white men," Journal of Urology, vol. 160, pp. 1734-1738, 1999.

[8] P. H. Gann, et al., "A prospective evaluation of plasma prostate-specific antigen for detection of prostatic cancer," Journal of the American Medical Association, vol. 273, pp. 289-294, 1995.

[9] P. Lodding, et al., "Characteristics of screening detected prostate cancer in men 50 to 66 years old with 3 to 4 ng./Ml. Prostate specific antigen," Journal of Urology, vol. 159, pp. 899-903, 1998.

[10] D. S. Smith, et al., "Longitudinal screening for prostate cancer with prostate-specific antigen," Journal of the American Medical Association, vol. 276, pp. 1309-1315, 1996.

[11] C. H. Harris, et al., "Prospective longitudinal evaluation of men with initial prostate specific antigen levels of $4.0 \mathrm{ng} . / \mathrm{ml}$. or less," Journal of Urology, vol. 157, pp. 1740-1743, 1997.

[12] J. A. Eastham, et al., "Variation of Serum Prostate-Specific Antigen Levels: An Evaluation of Yearto-Year Fluctuations," Journal of the American Medical Association, vol. 289, pp. 2695-2700, 2003.

[13] B. Carter, et al., "Prostate-specific antigen variability in men without prostate cancer: Effect of sampling interval on prostate-specific antigen velocity," Urology, vol. 45, pp. 591-596, 1995.

[14] R. L. Waterhouse and M. I. Resnick, "The use of transrectal prostatic ultrasonography in the evaluation of patients with prostatic carcinoma," Journal of Urology, vol. 141, pp. 233-239, 1989.

[15] L. T. Szabo, Diagnostic ultrasound imaging: inside out: Elsevier Academic Press 2004.

[16] K. K. Hodge, et al., "Ultrasound guided transrectal core biopsies of the palpably abnormal prostate," Journal of Urology, vol. 142, pp. 66-70, 1989.

[17] L. A. Eskew, et al., "Systematic 5 region prostate biopsy is superior to sextant method for diagnosing carcinoma of the prostate," Journal of Urology, vol. 157, pp. 199-203, 1997.

[18] Faran, Jr., "Sound Scattering by Solid Cylinders and Spheres," The Journal of the Acoustical Society of America, vol. 23, pp. 405-418, 1951.

[19] R. E. Baddour, et al., "High-frequency ultrasound scattering from microspheres and single cells," The Journal of the Acoustical Society of America, vol. 117, pp. 934-943, 2005.

[20] E. J. Feleppa and M. M. Yaremko, "Ultrasonic Tissue Characterization for Diagnosis and Monitoring," Engineering in medicine \& biology, vol. 6, pp. 18-26, 1987.

[21] F. L. Lizzi, "Theoretical framework for spectrum analysis in ultrasonic tissue characterization," vol. 73, p. 1366, 1983. 
[22] F. L. Lizzi, et al., "Theoretical framework for spectrum analysis in ultrasonic tissue characterization," The Journal of the Acoustical Society of America, vol. 73, pp. 1366-1373, 1983.

[23] F. L. Lizzi, et al., "Ultrasonic spectrum analysis for tissue evaluation," Pattern Recognition Letters, vol. 24, pp. 637-658, 2003.

[24] F. L. Lizzi, et al., "Statistics of ultrasonic spectral parameters for prostate and liver examinations," IEEE Transactions on Ultrasonics, Ferroelectrics, and Frequency Control, vol. 44, pp. 935-942, 1997.

[25] F. L. Lizzi, et al., "Relationship of Ultrasonic Spectral Parameters to Features of Tissue Microstructure," Ultrasonics, Ferroelectrics and Frequency Control, IEEE Transactions on, vol. 34, pp. 319-329, 1987.

[26] F. L. Lizzi, et al., "Comparison of theoretical scattering results and ultrasonic data from clinical liver examinations," Ultrasound in Medicine \& Biology, vol. 14, pp. 377-385, 1988.

[27] E. J. Feleppa, et al., "Ultrasonic spectral-parameter imaging of the prostate," International Journal of Imaging Systems and Technology, vol. 8, pp. 11-25, 1997.

[28] E. J. Feleppa, et al., "Diagnostic spectrum analysis in ophthalmology: A physical perspective," Ultrasound in Medicine and Biology, vol. 12, pp. 623-631, 1986.

[29] T. Noritomi, et al., "In vitro investigation of lymph node metastasis of colorectal cancer using ultrasonic spectral parameters," Ultrasound in Medicine and Biology, vol. 24, pp. 235-243, 1998.

[30] E. J. Feleppa, et al., "Typing of prostate tissue by ultrasonic spectrum analysis," Ultrasonics, Ferroelectrics and Frequency Control, IEEE Transactions on, vol. 43, pp. 609-619, 1996.

[31] E. J. Feleppa, et al., "Progress in two-dimensional and three-dimensional ultrasonic tissue-type imaging of the prostate based on spectrum analysis and nonlinear classifiers," Molecular Urology, vol. 3, pp. 303-311, 1999.

[32] E. J. Feleppa, et al., "Two-dimensional and three-dimensional tissue-type imaging of the prostate based on ultrasonic spectrum analysis and neural-network classification," Proceedings of SPIE The International Society for Optical Engineering, vol. 3982, pp. 152-160, 2000.

[33] W. Gnadt, et al., "Classification of prostate tissue using neural networks," pp. 3569-3572.

[34] E. J. Feleppa, et al., "New developments in tissue-type imaging (TTI) for guiding prostate biopsies and for planning and monitoring treatment of prostate cancer," in Ultrasonics Symposium, 2004 IEEE, pp. 834-837.

[35] G. J. Czarnota, "Ultrasound imaging of apoptosis: high-resolution non-invasive monitoring of programmed cell death in vitro, in situ and in vivo," vol. 81, pp. 520-527, 1999.

[36] M. C. Kolios, et al., "Ultrasonic spectral parameter characterization of apoptosis," Ultrasound in Medicine and Biology, vol. 28, pp. 589-597, 2002.

[37] G. J. Czarnota, et al., "Ultrasonic biomicroscopy of viable, dead and apoptotic cells," Ultrasound in Medicine and Biology, vol. 23, pp. 961-965, 1997.

[38] R. M. Vlad, et al., "Quantitative Ultrasound Characterization of Cancer Radiotherapy Effects In Vitro," International Journal of Radiation Oncology Biology Physics, vol. 72, pp. 1236-1243, 2008.

[39] R. M. Vlad, et al., "Quantitative ultrasound characterization of responses to radiotherapy in cancer mouse models," Clinical Cancer Research, vol. 15, pp. 2067-2075, 2009.

[40] R. M. Vlad, et al., "High-frequency ultrasound for monitoring changes in liver tissue during preservation," Physics in Medicine and Biology, vol. 50, pp. 197-213, 2005.

[41] Naum Papanicolau, "Conventional Frequency Ultrasound Detection of Tumor Responce In Vivo to Cancer Treatment Administration," MSc, Electrical and Computer Engineering, Ryerson Univeristy, Toronto, 2009.

[42] M. L. Oelze, et al., "Differentiation and characterization of rat mammary fibroadenomas and 4T1 mouse carcinomas using quantitative ultrasound imaging," IEEE Transactions on Medical Imaging, vol. 23, pp. 764-771, 2004. 
[43] M. L. Oelze, et al., "Ultrasonic quantification of the tissue microstructure of spontaneous mammary tumors in rats," in IEEE Ultrasonics Symposium, Munich, 2002, pp. 1369-1372.

[44] M. L. Oelze, et al., "Differentiation of tumor types in vivo by scatterer property estimates and parametric images using ultrasound backscatter," in IEEE Ultrasonics Symposium, Honolulu, 2003, pp. 1014-1017.

[45] F. Dong, et al., "Nonlinearity parameter for tissue-mimicking materials," Ultrasound in Medicine and Biology, vol. 25, pp. 831-838, 1999.

[46] D. Sage, et al., "MIJ," 1.3.6 ed: Biomedical Image Group (BIG), Ecole Polytechnique Fédérale de Lausanne (EPFL), Switzerland, 2009, pp. A Java package for bi-directional communication and data exchange from Matlab to ImageJ.

[47] Kitware. (Visualization Toolkit). Available: http://www.vtk.org/

[48] M. Frank, "Visualization Toolkit for C\# in Scope of ROTOR Project," Master of Science, Department of Computer Science and Engineering, University of West Bohemia, Pilsen, 2003.

[49] M. Frank. (2003, January 2009). VTK.NET. Available: http://graphics.zcu.cz/research/VTKforNET/download/index.html

[50] S. K. Alam, et al., "Prostate elastography - Preliminary in vivo results," 2005, pp. 339-345.

[51] P. Chaturvedi, et al., "Ultrasonic and elasticity imaging to model disease-induced changes in soft-tissue structure," Medical Image Analysis, vol. 2, pp. 325-338, 1998.

[52] J. Ophir, et al., "Elastography: Ultrasonic estimation and imaging of the elastic properties of tissues," Proceedings of the Institution of Mechanical Engineers, Part H: Journal of Engineering in Medicine, vol. 213, pp. 203-233, 1999.

[53] J. Ophir, et al., "Elastography: Ultrasonic imaging of tissue strain and elastic modulus in vivo," European Journal of Ultrasound, vol. 3, pp. 49-70, 1996.

[54] T. J. Hall, "AAPM/RSNA Physics Tutorial for Residents: Topics in US - Beyond the Basics: Elasticity Imaging with US," Radiographics, vol. 23, pp. 1657-1671, 2003.

[55] E. S. Burnside, et al., "Differentiating benign from malignant solid breast masses with US strain imaging," Radiology, vol. 245, pp. 401-410, 2007.

[56] A. Thomas, et al., "Real-time elastography - An advanced method of ultrasound: First results in 108 patients with breast lesions," Ultrasound in Obstetrics and Gynecology, vol. 28, pp. 335-340, 2006. 